\title{
A minor merger origin for stellar inner discs and rings in spiral galaxies ${ }^{\star}$
}

\author{
M. C. Eliche-Moral ${ }^{1}$, A. C. González-García ${ }^{2,3,4}$, M. Balcells ${ }^{3,4,5}$, \\ J. A. L. Aguerri ${ }^{3,4}$, J. Gallego ${ }^{1}$, J. Zamorano ${ }^{1}$, and M. Prieto ${ }^{3,4}$ \\ 1 Departamento de Astrofísica, Universidad Complutense de Madrid, 28040 Madrid, Spain \\ e-mail: mceliche@fis.ucm.es \\ 2 Dept. Física Teórica, Universidad Autónoma de Madrid, 28049 Madrid, Spain \\ 3 Instituto de Astrofísica de Canarias, C/ vía Láctea, 38200 La Laguna, Tenerife, Spain \\ 4 Departamento de Astrofísica, Universidad de La Laguna, 38200 La Laguna, Tenerife, Spain \\ 5 Isaac Newton Group of Telescopes, Apartado 321, 38700 Santa Cruz de La Palma, Canary Islands, Spain \\ Received 13 January 2011 / Accepted 19 May 2011
}

\section{ABSTRACT}

\begin{abstract}
Context. Observations have shown that inner discs and rings (IDs and IRs) are not preferably found in barred galaxies, which indicates that their formation may differ from that described by the traditional bar-origin scenario in many cases. In contrast, the role of minor mergers in producing these inner components (ICs), while often invoked, is still poorly understood.

Aims. We investigate the capability of minor mergers to trigger the formation of IDs and IRs in spiral galaxies through collisionless $N$-body simulations.

Methods. We run a battery of minor merger simulations in which both primary and secondary galaxies are modelled as disc-bulgehalo galaxies with realistic density ratios. Different orbits and mass ratios are considered, as well as two different models for the primary galaxy (a Sab or Sc). We then perform a detailed analysis of the morphology, structure, and kinematics of the ICs resulting from the minor merger.

Results. All the simulated minor mergers develop thin ICs out of satellite material, supported by rotation. A wide morphological zoo of ICs are obtained (including IDs, IRs, pseudo-rings, nested IDs, spiral patterns, and combinations of them), but all have structural and kinematical properties similar to those observed. The sizes of the resulting ICs are comparable to those observed in real galaxies with the adequate scaling. The existence of the resulting ICs can be deduced from the features that they imprint in the isophotal profiles and kinemetric maps of the final remnant, as in many real galaxies. Weak transitory oval distortions appear in the remnant centre in many cases, but none of them develops a noticeable bar. The realistic density ratios used in the present models ensure that the satellites experience more efficient orbital circularization and disruption than in previous studies. Combined with the disc resonances induced by the encounter, these processes produce highly aligned co- and counter-rotating ICs at the remnant centre.

Conclusions. Minor mergers are an efficient mechanism for forming rotationally-supported stellar ICs in spiral galaxies, without requiring either strong dissipation or the development of noticeable bars. The present models indicate that minor mergers can account for the existence of pure-stellar old ICs in unbarred galaxies, and suggest that their role must have been crucial in the formation of ICs and much more complex than just bar triggering.
\end{abstract}

Key words. galaxies: bulges - galaxies: evolution - galaxies: formation - galaxies: interactions - galaxies: structure galaxies: kinematics and dynamics

\section{Introduction}

Stellar IDs and IRs are found in at least one third of spiral galaxies (Erwin \& Sparke 2002; Falcón-Barroso et al. 2006, F06 hereafter). Usually masked by the light coming from brighter overlapping components, many of them have been detected through the peculiar features that these ICs imprint on the isophotes or the kinematic maps of the host galaxy (van den Bosch et al. 1994; Kormendy et al. 1994; Lauer et al. 1995; Scorza et al. 1998; Scorza \& van den Bosch 1998; Koprolin \& Zeilinger 2000; Rest et al. 2001; Erwin 2004; Carollo 2004; Lisker et al. 2006; Comerón et al. 2008; Morelli et al. 2010; Moiseev et al. 2010; Sil'Chenko 2010).

Stellar IDs are found in galaxies of all types, although they tend to reside in Sa-Sb's. Their radii span from some tens of parsecs to $\sim 2 \mathrm{kpc}$ in diameter. They exhibit red or blue colours,

* Figures 12-16 are available in electronic form at http://www . aanda.org usually similar to those of the host bulges, and harbour stellar populations with ages ranging from $\sim 1$ Gyr to $\sim 10$ Gyr (Franx \& Illingworth 1988; Bender 1990; van der Marel \& Franx 1993; Cinzano \& van der Marel 1994; Prieto et al. 2001; Carollo et al. 2002; Erwin \& Sparke 2002; Martel et al. 2002; Erwin \& Sparke 2003; Kormendy \& Kennicutt 2004; Morelli et al. 2004; Athanassoula 2005; Cappellari et al. 2007; Emsellem et al. 2007; Peletier et al. 2007). Inner star-forming IRs and pseudo-rings are detected in at least one fifth of all disc galaxies (Knapen 2005; Moiseev \& Bizyaev 2009; de Lapparent et al. 2011). However, because of the episodic nature of their star formation histories and their long-lived stable configurations, the total fraction of IRs including pure stellar (non star-forming) ones is expected to be much higher (Mazzuca et al. 2006; Sarzi et al. 2007).

These stellar ICs could be primordial features in the galaxy centres, established at an early epoch of rapid accretion of cold gas at high redshift (Bedregal et al. 2006). However, the dramatic decline in the star formation surface density in the 
discs in the past $\sim 8 \mathrm{Gyr}$ and the correlations found between the nuclear and disc global properties of spiral galaxies imply that there is an intertwined star formation history for bulges, discs, and ICs that spreads through time (Peletier \& Balcells 1996; Domínguez-Palmero et al. 2008; Domínguez-Palmero \& Balcells 2008). Owing to the large number of stellar IDs and IRs detected in barred galaxies, their origin has been traditionally associated with bar patterns in discs (Combes et al. 1992; Arnaboldi et al. 1995a; van den Bosch \& Emsellem 1998; Erwin \& Sparke 2002; Regan \& Teuben 2003; Böker et al. 2008; de Lorenzo-Cáceres et al. 2008; Comerón et al. 2010). This has been corroborated by numerical simulations, which have shown that the dynamical resonances induced by bars easily produce the rings surrounding them. Moreover, the bars induce strong gas inflows to the galaxy centre, which settle in circular orbits, inducing star formation that can result in an ID (Norman et al. 1996; Athanassoula et al. 2009b,a; Comerón et al. 2010). Other bar-related scenarios propose that IDs can also be the relics of diluted nested bars or episodes of starbursts in rings that have shrunk in radius to the galaxy centre over time (Combes et al. 1992; Regan \& Teuben 2003; Sil'Chenko \& Smirnova 2010).

Nevertheless, observations indicate that stellar IDs and IRs do not exist primarily in barred galaxies, but are at least as frequent in non-barred early-type hosts as in barred ones (Emsellem et al. 2004; Falcón-Barroso et al. 2004; Knapen 2005; Knapen et al. 2006; Sarzi et al. 2006; Comerón et al. 2010). IRs are easily produced by unstable gas-rich discs in numerical simulations (Aumer et al. 2010), but IDs are structures that are more difficult to reproduce spontaneously (Thakar et al. 1997). Hence, we need to consider other mechanisms capable of triggering disc resonances besides bars to explain the existence of stellar IDs and IRs in unbarred galaxies. One of the main alternatives is gas infall, but simulations indicate that this mechanism is efficient in producing IRs, but not IDs (Thakar \& Ryden 1998; Naab et al. 2006).

Another possible driver is merging, as suggested by the existence of many ICs exhibiting strong misalignments or distorted morphologies and/or kinematics with respect to the host disc (see, e.g., Okumura et al. 1994; Arnaboldi et al. 1995b; Buta \& Combes 1996; Barnes \& Hernquist 1996; Knapen et al. 2004; Reshetnikov et al. 2005; Mazzuca et al. 2006; Sil'Chenko \& Moiseev 2006; Chilingarian et al. 2009; Faúndez-Abans et al. 2009; Moiseev \& Bizyaev 2009; Brosch et al. 2010). This scenario is supported by numerical simulations, which have proven that major mergers can drive the formation of kinematicallydecoupled ICs analogous to the ones found in E-S0 galaxies (Hernquist \& Barnes 1991; Balcells \& González 1998; Bendo \& Barnes 2000; Barnes 2001; Jesseit et al. 2007; Di Matteo et al. 2008). Since massive E-S0 galaxies have experienced at least one major merger since $z \sim 1$ (González-García et al. 2009; López-Sanjuan et al. 2009; Eliche-Moral et al. 2010a,b), this means that the existence of ICs in E-SO's can be satisfactorily explained by this process. However, they cannot account for the ICs found in unbarred $\mathrm{Sa}-\mathrm{Sb}$ galaxies, as the remnants resulting from major mergers are E-S0's for typical amounts of gas (Barnes \& Hernquist 1996; Bournaud et al. 2004, 2005b; Naab \& Burkert 2003).

The straightforward alternative to major mergers is minor merging. Minor mergers induce dynamical resonances and oval distortions in discs easily and trigger a smooth growth of the pre-existing galaxy bulge (see Bertola et al. 1999; Pizzella et al. 2002; Eliche-Moral et al. 2006; Sil'Chenko \& Moiseev 2006; Brosch et al. 2010). Although the effects of minor mergers on bulge and disc growth and on the satellites have been extensively studied (e.g., Hopkins et al. 2010; Henriques \& Thomas 2010; Tapia et al. 2010a,b; Bartošková et al. 2011; Ebrova et al. 2011), little attention has been devoted to studying specifically their ability to induce the formation of dynamically-cold ICs in galaxies.

One of the earliest studies dealing with this topic was carried out by Elmegreen et al. (1992), who performed numerical simulations of ring-companion interactions to analyse the effects of the minor mergers on pre-existing outer rings. Later, Thakar and collaborators investigated the formation of counter-rotating discs in spiral galaxies through retrograde mergers of gas-rich dwarfs onto discs (Thakar \& Ryden 1996, 1998; Thakar et al. 1997). They found that, although counter-rotating thin gaseous discs were formed during minor mergers, the obtained sizes were comparable only to the largest observational cases. The IDs formed did not have exponential profiles either and were highly unstable, quickly becoming IRs. This led these authors to conclude that, in order to form a normal counter-rotating disc, "there must be either little or no pre-existing prograde gas in the primary galaxy, or its dissipative influence must be offset by significant star formation activity". Aguerri et al. (2001) performed collisionless $N$-body simulations to test the growth of bulges after the accretion of dense spheroidal satellites. Their undisrupted dense satellite cores sank to the galaxy centre, producing kinematically-decoupled components in the remnants that were not supported by rotation.

Eliche-Moral et al. (2006, EM06 hereafter) studied the effects of the accretion onto disc galaxies using satellites that themselves comprised a disc, a bulge, and a dark halo. More importantly, authors verified that the relative mass densities of the primary and secondary galaxies were realistic by checking that both models lie on the Tully-Fisher relation. This was an improvement over previous studies of mass buildup via accretion, given that the disruption of the satellite, the radius of deposition of satellite material, and the dynamical heating of the primary, all depend on the tidal fields, which scale with the relative densities of the two galaxies. EM06 reported the formation of dynamically-cold stellar structures in the centre of remnants, that consisted of disrupted satellite material. However, that paper was based on the bulge growth driven by the minor merger, hence no analysis or description of the ICs was performed. We therefore extended the simulations of EM06, sampling different orbits and initial conditions and using $\sim 3$ times more particles, to carry out an exhaustive study of the morphology and kinematics of the ICs resulting from different minor mergers. We simulated collisionless cases, as recent studies have demonstrated that dissipative components are not uniquely responsible for the formation and shaping of kinematically-decoupled ICs during major mergers (although gas makes them more axisymmetric and can induce recent star formation in them, see Jesseit et al. 2007; Di Matteo et al. 2008).

The present models demonstrate the capability of minor mergers to induce the formation of a wide morphological zoo of thin stellar ICs in the galaxy centres, with structural and kinematical properties analogous to those harboured by real spiral galaxies. The novelty of these models is two-fold: first, all the resulting ICs are made out of disrupted satellite material (whereas they come from resonances in the parent disc in previous studies) and, secondly, dissipative effects and strong bars are not essential to form these dynamically-cold ICs.

The paper is structured as follows. We briefly describe the models in Sect. 2. The formed ICs are analysed geometrically, photometrically, and kinematically in Sect. 3. In Sect. 4, we perform a qualitative comparison of the ICs resulting in the models 
Table 1. Number of particles used in the models.

\begin{tabular}{lccccccc}
\hline \hline & \multicolumn{7}{c}{ Number of particles $\left(/ 10^{3}\right)$} \\
\hline Experiment type & Total & D1 & B1 & H1 & D2 & B2 & H2 \\
$(1)$ & $(2)$ & $(3)$ & $(4)$ & $(5)$ & $(6)$ & $(7)$ & $(8)$ \\
\hline Big bulge & 185 & 40 & 10 & 90 & 10 & 5 & 30 \\
Small bulge & 415 & 60 & 10 & 300 & - & - & - \\
\hline
\end{tabular}

Notes. Columns: (1) experiment type depending on the used primary galaxy model (big or small bulge); (2) total particle number; (3) number of primary disc particles; (4) number of primary bulge particles; (5) number of primary halo particles; (6) number of satellite disc particles; (7) number of satellite bulge particles; (8) number of satellite halo particles.

with those detected in real spiral galaxies. Model limitations are described in Sect. 5. Our discussion can be found in Sect. 6. A brief summary of the results and some conclusions are finally addressed in Sect. 7. The physical magnitudes are provided in units of the simulation throughout the paper, although scaling to real galaxies is straightforward assuming the scalings described in Sect. 2.

\section{The models}

We extended the set of collisionless $N$-body simulations of minor mergers onto disc galaxies described in EM06, now using longer pericentres and different initial disc galaxies. The outcome of the satellite material of a final set of 12 collisionless models was then analysed (six of them come from EM06). Ten of these experiments were run using a disc galaxy with a prominent bulge as primary galaxy (equivalent to a $\mathrm{Sa}-\mathrm{Sb}$ galaxy), while in another two a primary with a smaller bulge (similar to a Sc) was used to investigate the influence of the primary bulge in the outcome of the accretion.

All the galaxies in the simulations (primary galaxies and satellites) have an initial bulge-disc-halo structure. The primary galaxy models were built using the Galact ICS code (Kuijken \& Dubinski 1995), including an exponential disc component (Shu 1969), a King bulge (King 1966), and a dark halo built following an Evans profile (Kuijken \& Dubinski 1994). The discs of both primary galaxy models follow an exponential surface density profile both radially and vertically, and were allowed to relax in isolation for about ten disc dynamical times prior to placing them in orbit for the merger simulations. No relevant resonant structures appear in the disc. The primary galaxy with a large bulge matches the Milky Way (MW) when the units of length, velocity, and mass are $R=4.5 \mathrm{kpc}, v=220 \mathrm{~km} \mathrm{~s}^{-1}$, and $M=5.1 \times 10^{10} M_{\odot}$, respectively. In this case, the corresponding time unit is 20.5 Myr. The primary galaxy with a small bulge matches NGC 253 using the units of length, velocity, and mass $R=6.8 \mathrm{kpc}, v=510 \mathrm{~km} \mathrm{~s}^{-1}$, and $M=2.6 \times 10^{11} M_{\odot}$, implying a time unit of $11.7 \mathrm{Myr}$. These values, especially when using an appropriate $M_{\text {lum }} / L$ ratio, yield mass-to-light ratio values close to observations $(M / L \sim 10)$. Tables 1 and 2 summarize the main characteristics of the two relaxed galaxy models used to represent the primary galaxy. For the remainder of the article, we use model units in all the figures, a conversion to physical units is easily achieved through the above correspondences.

Satellites are scaled replicas of the primary galaxy model with a big bulge in all the experiments. A physically-motivated size-mass scaling was used to ensure that the primary-to-satellite density ratios are realistic, forcing both galaxies to obey the Tully-Fisher relation (Tully \& Fisher 1977, consult EM06). In
Table 3, we list the characteristic masses and sizes of the satellites for each merger experiment. The luminous mass ratios between the primary galaxy and the satellite considered were 1:6, $1: 9$, and 1:18. In the models with large primary bulges, the primary and satellite are scaled replicas, so the luminous mass ratio of the encounter is equivalent to its total mass ratio. However, as mentioned above, the satellites in the models with small primary bulges are scaled versions of the large primary bulge model, in order to analyse the influence of the primary bulge structure on the outcome of the accretion. Satellites were also relaxed in isolation prior to the merging simulation.

The initial separation of both galaxies was 15 primary disc scale-lengths in all the experiments. To avoid a perfect spin-orbit coupling, the initial inclinations between the orbital plane and the galactic planes of the primary and the satellite galaxies were fixed to $30^{\circ}$ in direct orbits and to $150^{\circ}$ in retrograde orbits, respectively. All satellites also have an azimuthal angle $\phi=90^{\circ}$. For each satellite mass, pericentre distance, and model of the primary galaxy considered, a direct and a retrograde orbit were computed. Initial orbits were elliptical with pericentres equal to one or eight disc scale-lengths, depending on whether we considered a short or a long pericentre orbit. Relative velocities at the first pericentre passage oscillate in the range $440-650 \mathrm{~km} \mathrm{~s}^{-1}$, considering the scalings commented above.

In Table 3, we include the orbital parameters of each merging experiment. As satellite models are scaled-down versions of the primary model with a large bulge and exhibit different mass ratios with the same number of particles, this renders different mass particles for the different components in each experiment. The largest mass contrast, and thus the higher two-body errors, are expected in the models with a total mass ratio of $1: 18$, where a primary halo particle is ten times more massive than the bulge particles of the satellite. This extreme ratio is well below the limits explored earlier in different simulations using a similar set of initial models, so the kinematics of the inner regions of the remnant are much more reliably sampled in the present models than previously (Balcells \& González 1998; González-García \& Balcells 2005; EM06). The difference in the number of particles for the haloes hosting a large and a small bulge accounts for two facts. On the one hand, a bulge-less disc model stable to bar distortions requires a concentrated halo (see, e.g., González-García $\&$ Balcells 2005). On the other hand, the masses of the halo and bulge particles need to be of the same order to ensure that there are small two-body errors. These two constraints require the models with a small bulge to have such a high number of halo particles. As we are interested in the physics of the inner regions of the remnants, we attempted to find a balance between the accuracy gained by using a larger number of particles and the economy of resources in building new stable models for each satellite.

The evolution of the new models was computed using the GADGET-2 code (Springel et al. 2001; Springel 2005). We used a softening of $\varepsilon=0.02$ in model units and an opening angle of $\theta=0.6$. Considering this tolerance parameter and applying quadrupole-moment corrections, the code computes forces to within $1 \%$ of those given by a direct summation and preserves the total energy to better than $0.1 \%$. We evolved all models for $\sim 4$ halo crossing times beyond a full merger to allow the final remnants to reach a quasi-equilibrium state with a good conservation of energy in all runs. Times to full merger and total run times of each experiment are also indicated in Table 3 in simulation units. The initial $B / D$ ratios of the main galaxy increase after the merger to $B / D \sim 0.6$ in the cases of large bulges and $B / D \sim 0.2$ for small bulges. This means that the remnants of 
Table 2. Initial parameters of the primary galaxy models.

\begin{tabular}{lccccccc}
\hline \hline Experiment type & $\mathcal{M}_{\mathrm{T}, 1}$ & $\mathcal{M}_{\mathrm{B}, 1} / \mathcal{M}_{\mathrm{D}, 1}$ & $\mathcal{M}_{\mathrm{Dark}, 1} / \mathcal{M}_{\mathrm{L}, 1}$ & $h_{\mathrm{D}, 1}$ & $r_{\mathrm{B}, 1} / h_{\mathrm{D}, 1}$ & $h_{95 \%, 1} / h_{\mathrm{D}, 1}$ & $z_{\mathrm{D}, 1} / h_{\mathrm{D}, 1}$ \\
$(1)$ & $(2)$ & $(3)$ & $(4)$ & $(5)$ & $(6)$ & $(7)$ & $(8)$ \\
\hline Big primary bulge & 6.40 & 0.51 & 4.16 & 1.00 & 0.20 & 3.7 & 0.11 \\
Small primary bulge & 0.99 & 0.08 & 6.98 & 0.39 & 0.16 & 3.8 & 0.06 \\
\hline
\end{tabular}

Notes. Columns: (1) experiment type depending on the primary galaxy model used (big or small bulge); (2) total primary mass (simulation units); (3) bulge-to-disc mass ratio of primary galaxy; (4) dark-to-luminous mass ratio of primary galaxy; (5) radial disc scale-length of the primary galaxy; (6) ratio of the effective radius of primary bulge to primary disc scale-length; (7) ratio of the radius of the shell containing $95 \%$ of luminous material in the primary galaxy to its disc scale-length; (8) ratio of the vertical to radial disc scale-lengths of the primary galaxy.

Table 3. Orbital and scaling parameters of each merger experiment.

\begin{tabular}{|c|c|c|c|c|c|c|c|c|}
\hline $\begin{array}{l}\text { Model code } \\
\text { (1) }\end{array}$ & $\begin{array}{c}\text { Code in EM06 } \\
\text { (2) }\end{array}$ & $\begin{array}{c}\text { Primary bulge } \\
\text { (3) }\end{array}$ & $\begin{array}{c}\mathcal{M}_{\mathrm{L}, \text { Sat }} / \mathcal{M}_{\mathrm{L}, \text { Prim }} \\
\text { (4) }\end{array}$ & $\begin{array}{c}R_{\text {Sat }} / R_{\text {Prim }} \\
\quad \text { (5) }\end{array}$ & $\begin{array}{c}R_{\text {pericentre }} / h_{\mathrm{D}, 1} \\
\text { (6) }\end{array}$ & $\begin{array}{c}\theta_{\text {Prim }} \\
(7)\end{array}$ & $\begin{array}{l}t_{\text {full merger }} \\
\text { (8) }\end{array}$ & $\begin{array}{l}t_{\text {total }} \\
(9)\end{array}$ \\
\hline (a) M6 Ps Db & M2TF35D & Big (b) & 1:6 (M6) & 0.46 & $0.73(\mathrm{Ps})$ & 30 (D) & $\sim 72$ & 100 \\
\hline (b) M6 Ps Rb & M2R & Big (b) & 1:6 (M6) & 0.46 & 0.73 (Ps) & $150(\mathrm{R})$ & $\sim 80$ & 100 \\
\hline (c) M6 Pl Db & - & Big (b) & 1:6 (M6) & 0.46 & $8.25(\mathrm{Pl})$ & 30 (D) & $\sim 93$ & 144 \\
\hline (d) M6 Pl Rb & - & Big (b) & 1:6 (M6) & 0.46 & $8.25(\mathrm{Pl})$ & $150(\mathrm{R})$ & $\sim 110$ & 144 \\
\hline (e) M6 Ps Ds & - & Small (s) & 1:6 (M6) & 0.25 & 0.87 (Ps) & 30 (D) & $\sim 40$ & 62 \\
\hline (f) M6 Ps Rs & - & Small (s) & 1:6 (M6) & 0.25 & 0.87 (Ps) & $150(\mathrm{R})$ & $\sim 44$ & 72 \\
\hline (g) M9 Ps Db & M3TF35D & Big (b) & 1:9 (M9) & 0.39 & 0.79 (Ps) & 30 (D) & $\sim 80$ & 100 \\
\hline (h) M9 Ps Rb & M3R & Big (b) & 1:9 (M9) & 0.39 & 0.79 (Ps) & $150(\mathrm{R})$ & $\sim 87$ & 100 \\
\hline (i) M18 Ps Db & M6TF35D & Big (b) & 1:18 (M18) & 0.28 & 0.86 (Ps) & 30 (D) & $\sim 116$ & 122 \\
\hline (j) M18 Ps Rb & M6R & Big (b) & 1:18 (M18) & 0.28 & 0.86 (Ps) & $150(\mathrm{R})$ & $\sim 142$ & 154 \\
\hline (k) M18 Pl Db & - & Big (b) & 1:18 (M18) & 0.28 & $8.19(\mathrm{Pl})$ & 30 (D) & $\sim 225$ & 260 \\
\hline (1) M18 Pl Rb & - & Big (b) & 1:18 (M18) & 0.28 & $8.19(\mathrm{Pl})$ & $150(\mathrm{R})$ & $\sim 285$ & 340 \\
\hline
\end{tabular}

Notes. Columns: (1) Model code: $\mathrm{M} m \mathrm{P}[1 / \mathrm{s}][\mathrm{D} / \mathrm{R}][\mathrm{b} / \mathrm{s}]$, where $m$ indicates the bulge-to-satellite mass ratio $(m=6,9$, or 18 for models with luminous mass ratios equal to $1: 6,1: 9,1: 18$, respectively), "Pl" refers to long pericentre and "Ps" to short pericentre, "D" indicates direct orbits and "R" retrograde orbits), and the final letter ("b" or "s") indicates whether the primary galaxy had a large or a small bulge. The letter in parentheses helps us to identify each model quickly in the forthcoming figures; (2) model code in EM06, for those models that were already presented in that paper; (3) primary galaxy model used in the experiment (big or small primary bulge, see Table 1); (4) luminous mass ratio between satellite and primary galaxy; (5) ratio of the luminous half-mass radii of the satellite to the primary galaxy; (6) first pericentre distance of the orbit, in units of the primary disc scale-length; (7) initial angle between the orbital momentum and the primary disc spin. This angle determines whether the orbit is prograde (direct) or retrograde; (8) approximate time of full merger, in simulation units; (9) total run time of each experiment, in simulation units.

the experiments starting with a Sb primary galaxy have become S0-Sa's, while the Sc primary galaxies have been transformed into Sb-Sbc's after the minor merger (see Graham 2001).

We refer to each model throughout the paper according to the following code: $\mathrm{M} m \mathrm{P}[1 / \mathrm{s}][\mathrm{D} / \mathrm{R}][\mathrm{b} / \mathrm{s}]$, where $m$ indicates the bulge-to-satellite mass ratio ( $m=6,9$, or 18 for models with luminous mass ratios equal to $1: 6,1: 9,1: 18$, respectively), "Pl" indicates long pericentre and "Ps" short pericentre, "D" or "R" describes the orbit ("D" for direct and "R" for retrograde), and the final "b" or "s" letter indicates if the primary galaxy had a big or a small bulge (see Table 3 ).

\section{Results}

In EM06, we reported the formation of inner dynamically-cold components in minor merger experiments, but we analysed the resulting ICs neither structurally nor kinematically. In the next few sections, we perform a detailed analysis of the co- and counter-rotating ICs formed in the models described in Sect. 2.

\subsection{Formation of ICs}

In Fig. 1, we show the disruption experienced by the satellite in model M18PsDb (model $\mathrm{i}$ in Table 3) as an example of the time evolution of the luminous surface density of the satellite material during the last moments of the encounter. Although the different initial conditions affect the global shape, size, and even the number of components in these inner structures, the final structure in all the experiments resembles a central vertically thin torus or disc (depending on whether the satellite material reaches the remnant centre or not), embedded in a more extended flat component, similar to a disc. In the model plotted in the figure, the toroidal structure corresponds to the central ring visible at $R \sim 0.5$, while the outer disc corresponds to the low density structure that extends to $R \sim 1-1.5$. The morphologies of the ICs formed in each experiment are described in detail in Sect. 3.2.3.

Figure 1 also shows that the outer and inner regions of the structure resulting from the satellite disruption are built up at different epochs during the minor merger. The more weakly bounded particles of the satellite (i.e., those from the disc) are disrupted earlier in the interaction. During the first pericentre cross of the orbit, its outer shells are removed from the satellite by the primary galaxy tidal field, producing the outer structure of the formed IC (as observed in the first half of snapshots in Fig. 1). The core of the satellite takes more time to experience a noticeable disruption. Its material is deposited at inner radii during the last stages of the encounter.

\subsection{Geometrical and kinematical characterization of ICs}

We describe the geometry and structure of these ICs in terms of their radial and vertical surface brightness profiles, and analyse 

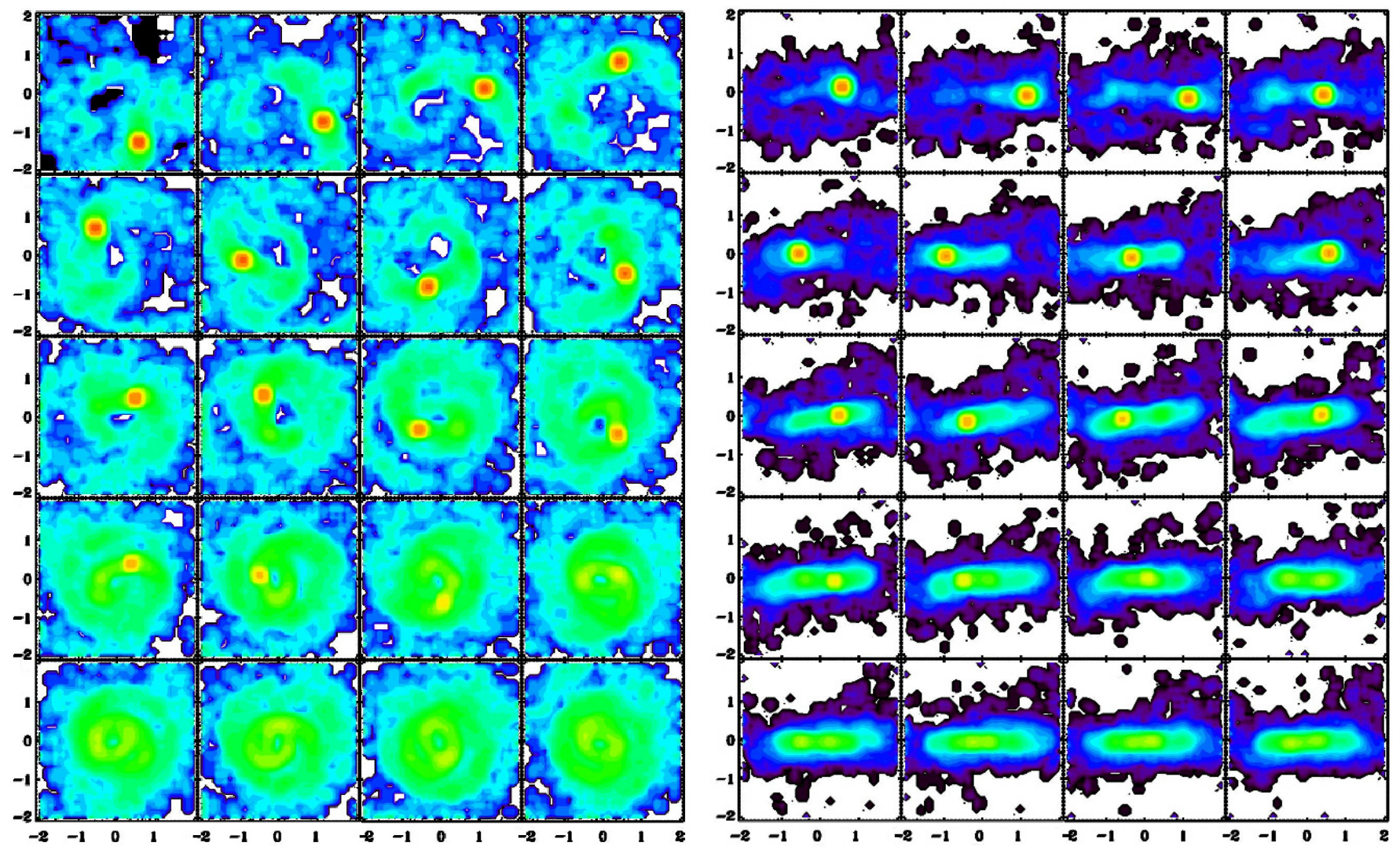

Fig. 1. Time evolution of the luminous surface density of the satellite material during the last moments of experiment M18PsDb (experiment "i" in Table 3). Face-on and edge-on views centred on the initial primary galaxy are plotted (left and right panels, respectively). Snapshots corresponding to times from 101 to 120 are shown from up-to-down and left-to-right of each figure, using a time step equal to one. If the primary galaxy is scaled to the MW (see Sect. 2), the total time period represented corresponds to $\sim 0.5 \mathrm{Gyr}$. A rainbow colour palette is used to represent different surface density levels in a logarithmic scale, with redder colours indicating higher values. Spatial scales in both axes are provided in simulation units. The disrupted satellite material is finally deposited in the remnant centre forming an IR (in yellow) embedded in a more extended ID (in green). The IR rotates in the same direction as the primary disc material.

their misalignment with respect to the galaxy plane of the final remnant and their kinematics.

\subsubsection{Radial distribution of ICs in the remnants}

Figure 2 shows the radial surface density profiles of luminous material initially belonging to the satellite, to its disc, and to its bulge in the final remnants of all the experiments. In general, the inner structure is basically composed of satellite bulge particles (typically, at $R \lesssim h_{\mathrm{D}, 1}(t=0)$ ), while the outer parts are controlled by the surface density of satellite disc particles. However, there is no sharp transition between the two radial ranges where each satellite component dominates.

We fitted one exponential disc or a combination of two nested ones to the surface density profiles of the satellite stellar material in each remnant (represented by two solid black straight lines in each panel). We assume that the satellite material described by each exponential radial profile defines an independent structure inside the global IC formed in the remnant centre out of disrupted satellite material. In the fits, we rejected the central regions of the density profiles to avoid the particular features exhibited by each distribution in the centre, such as undisrupted satellite cores (as in M6Ps[D/R]s, models e and f), holes (as in $\mathrm{M} 18 \mathrm{P}[\mathrm{l} / \mathrm{s}] \mathrm{Db}$, models $\mathrm{i}$ and $\mathrm{k}$ ), and smooth-length effects. The resulting fits and their residuals are plotted in the corresponding panels and sub-panels of Fig. 2.
Most of the resulting ICs can be closely approximated by one exponential radial profile or by the addition of two. However, we note that the independent ICs identified by each fitted exponential radial profile are shaped with material of both satellite components: from the bulge and the disc. Only in the experiments with a small primary bulge, the satellite bulge ends undisrupted in the remnant centre (see the profiles corresponding to the satellite bulge particles in M6PsDs and M6PsRs, frames e and $f$ in the figure). In these models, the outer shells of satellite material configure an extended ID structure hosting the undisrupted satellite core.

To delimit the radial extent of the ICs characterized by a unique exponential profile, we assumed that, in the case that only one exponential profile is required to explain the whole radial structure, this IC extends to the radius at which the fitted surface density is equal to $1 / 10$ of its central value as extrapolated from the fit. When the structure is more accurately described by two nested exponential profiles, the innermost one is considered to extend to the radius where the outer one starts to dominate the global fit. The outer one will extend from this radius to that at which its surface density drops to $1 / 10$ of its central value. The radial extent of each radial component resulting from these criteria is marked in Fig. 2.

Summarizing, all the simulated minor mergers produce complex extended ICs in the remnants consisting of disrupted satellite material, with radial surface density profiles that can be closely described by one or two nested exponential profiles. 

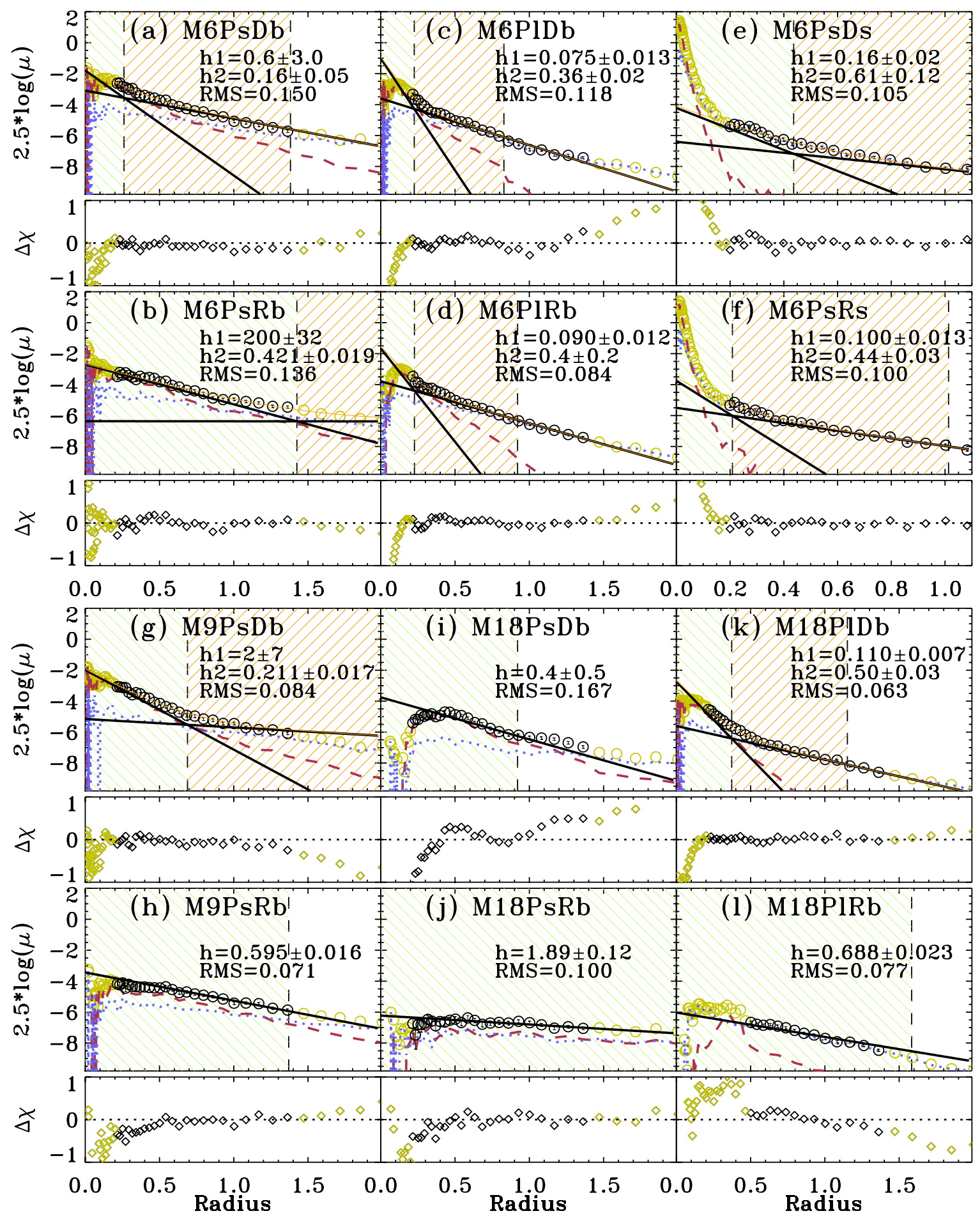

Fig. 2. Radial surface brightness profiles of disrupted satellite material in the final remnants of all the models. The plotted physical magnitudes are provided in simulation units. The letters identifying each panel are those used in Table 3. Circles: all the stars originally belonging to the satellite. Red dashed lines: satellite bulge stars. Blue dotted lines: satellite disc stars. Black solid straight lines: exponential fits to the radial density profiles considering all the satellite stars. The data points plotted in green have been excluded from the fits. In the panels corresponding to remnants that are more accurately fitted with two exponential discs, the two scale-lengths are shown in the frame. When just one exponential disc provides a better fit to the global satellite stellar profile than two nested ones, only one scale-length is provided. The root mean square of the global fit is also shown in each remnant. Orange light solid curved lines: total fit to the radial surface density profiles in those cases that are more accurately fitted by two exponential discs. Vertical dashed lines: extent of each IC characterized by each fitted exponential radial profile, as defined in the text. The radial extension of the ICs is indicated using a shaded background with a different colour for the different ICs identified in each model (green for the innermost ones and orange for the outer ones). Sub-panels: relative residuals of the fits as a function of the radial position. The models with small primary bulges obey a different scaling to the rest of models (see Sect. 2). Therefore, the simulation units in these two experiments differ from those in the others, as observed in Figs. 2-4. 

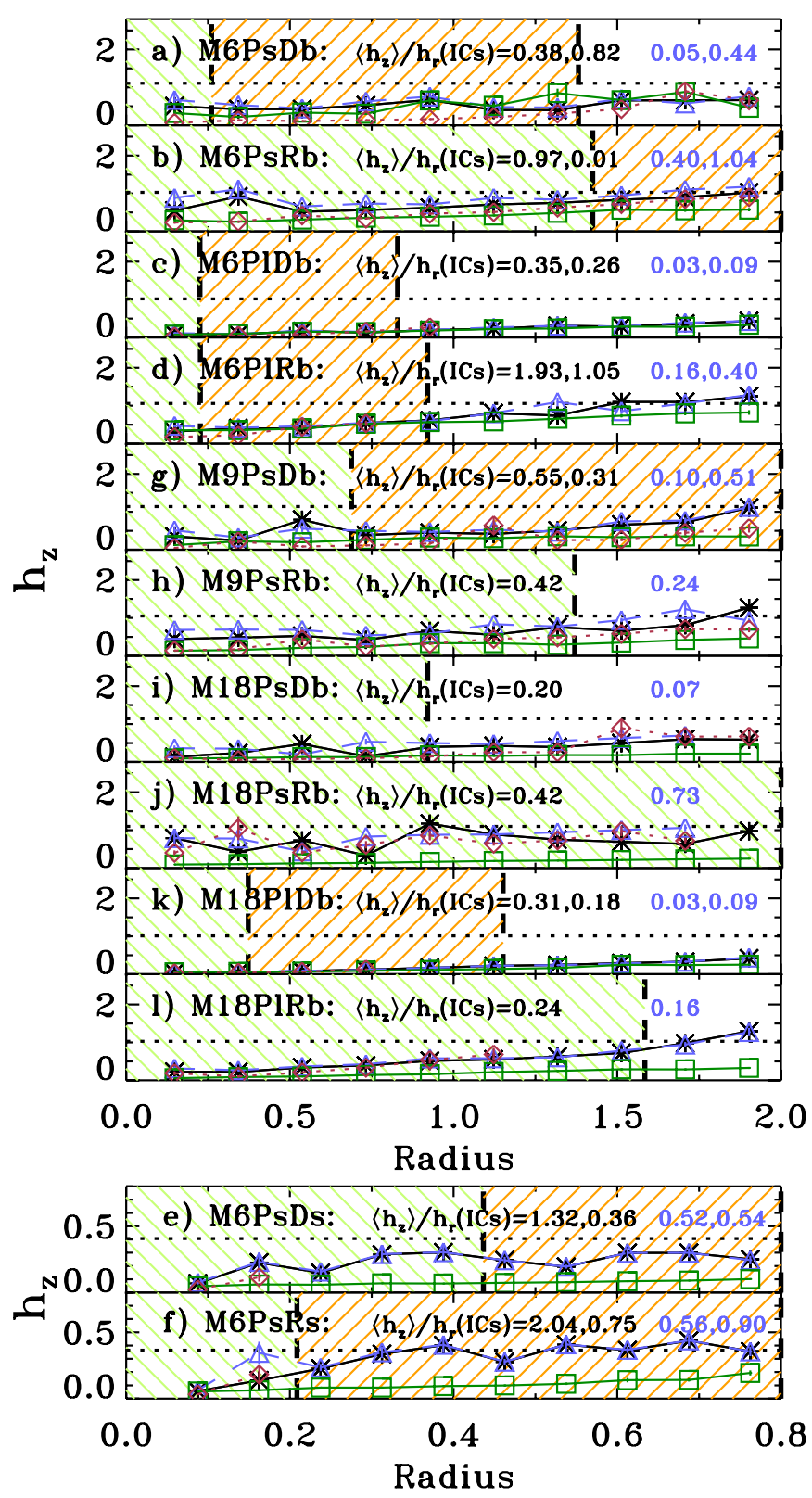

Fig. 3. Vertical exponential scale-lengths of material in the final remnants as a function of radius. The plotted physical magnitudes are provided in simulation units. The letters identifying each panel are those used in Table 3 for each model. Triangles: material originally from the satellite disc. Diamonds: from the satellite bulge. Asterisks: stellar material originally in the satellite. Squares: from the original primary disc. Horizontal dotted line: scale-length of the final remnant disc, marked as a reference. Vertical dashed lines: radial extent of each IC characterized by a unique exponential profile, according to Fig. 2. The ratio of the vertical to the radial scale-lengths of each $\mathrm{IC}$ is indicated in black characters (one number per radial exponential ICs). The ratio of the scale-height of each IC to the radial scale-length of the final remnant disc is also indicated in blue characters at the top right of each panel.

\subsubsection{Vertical distribution of ICs in the remnants}

An IC with a radial exponential profile can correspond to an ID or to a bulge with a Sérsic index $n=1$ (i.e., a pseudobulge). The difference between all these components arise in the vertical thickness of the component, as compared to its own radial scale-length and to the one of the host disc. Following
Sil'Chenko et al. (2011), a ratio of scale-length to scale-height of about 3 is a reasonable dividing line between spheroids and discs. Hence, we estimated the ratio of the vertical to the radial scale-lengths of the formed ICs in each remnant, as well as the ratio of their scale-heights to the radial scale-length of the remnant discs, to identify them as IDs (thin or thick) or pseudobulges.

Figure 3 presents the characteristic vertical scale-lengths $h_{z}$ at different radial positions of the disc, bulge, and all luminous material originally belonging to the satellite in the final remnants. The scale-lengths were derived from exponential fits to the vertical density profiles at each radius. Only the radial positions with enough particles to ensure a vertical density profile of $S / N>50$ were considered (typically, $R \lesssim 2 h_{\mathrm{D} \text {,Primary }}$ ). The fitting errors are on average $\lesssim 10 \%$ for each estimate. The final scale-length of the disc remnant is marked in each panel as a reference (horizontal dotted lines). We also indicated the radial extent of each IC, according to the definition adopted in Sect. 3.2.1.

We note that the IC made out of satellite material has scaleheights smaller than the radial scale-length of the original primary disc along the whole considered radial range in all the models, although it is vertically wider than the structure made out of primary disc material at all radii in all the remnants (compare asterisks and squares in Fig. 3). Hence, although the primary disc has been heated by the satellite accretion, it is thin compared to the IC. Therefore, the satellite material contributes to a structure thicker than the main remnant disc. This result is consistent with the structures of disc galaxies resulting from $\Lambda C D M$ cosmological simulations, where the disrupted satellites contribute to the buildup of the thick disc of the galaxy (Abadi et al. 2003). In general, the scale-height of the structure made of disrupted satellite material increases with radial position, meaning that these structures are flared (see asterisks in the figure).

The material of the disrupted satellite bulge tends to have smaller scale-heights than that of the disrupted satellite disc, but the different disruption epochs of both components does not seem to affect their final vertical distributions at a given radial position, as the scale-heights of both distributions are similar at each radius (compare red diamonds and blue triangles in the figure).

In Fig. 3, we also show the ratio of the vertical to radial scale-lengths of each IC (numbers in black characters in each frame). Nearly half the innermost ICs formed in the models with big primary bulges are thin, as they exhibit ratios typically below $\sim 0.4$. This means that these innermost components are IDs, according to the Sil'Chenko et al. (2011) criterion. Other cases exceed the limiting value proposed by these authors (as models M6P[s/l]Rb, panels $b$ and $d$ in the figure), meaning that these ICs would be classified as pseudo-bulges in the case that the original primary galaxies lacked a large central component. As this is not the case (they had a massive central bulge), the ICs formed in these models are embedded in the pre-existing bulge or the galaxy thick disc. We also have some questionable cases with vertical-to-radial scale-lengths near the limiting value for distinguishing between IDs and pseudobulges, as models M[9/18]PsRb (panels $h$ and $j$ in the figure).

The ratios of the scale-heights of these innermost components to the radial scale-length of the remnant disc indicate that the majority of these innermost ICs have ratios smaller than 0.4 (see the numbers in blue characters at the top right of each panel in the figure). This means that these structures are buried in the final remnant disc. The models with small primary bulges (panels e and $\mathrm{f}$ in the figure) exhibit large scale-heights at the centre, because the satellite core sinks to the remnant centre 


\section{a) M6PsDb: Nested IDs}
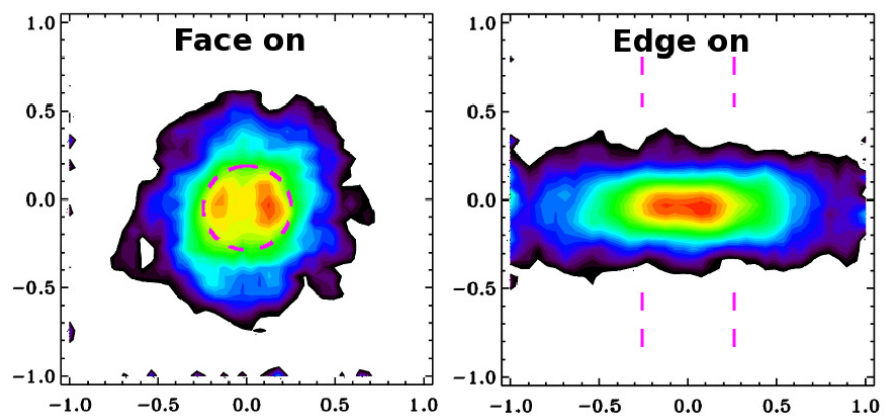

c) M6PIDb: Nested IDs
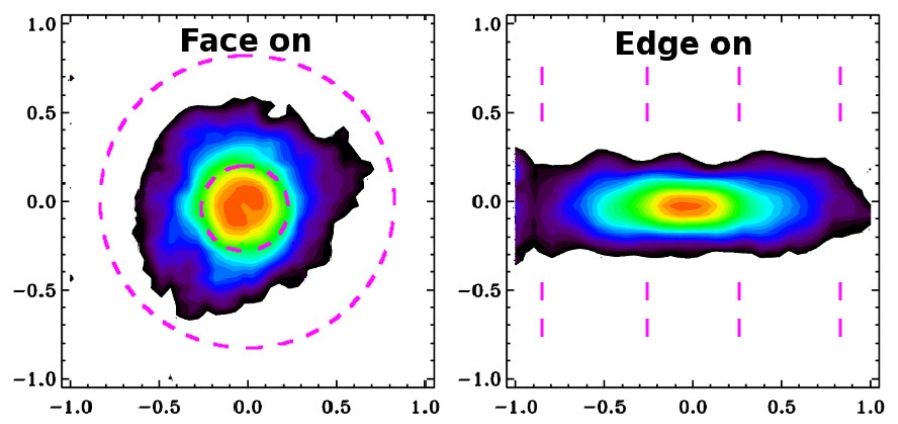

e) M6PsDs: Bulge + ID
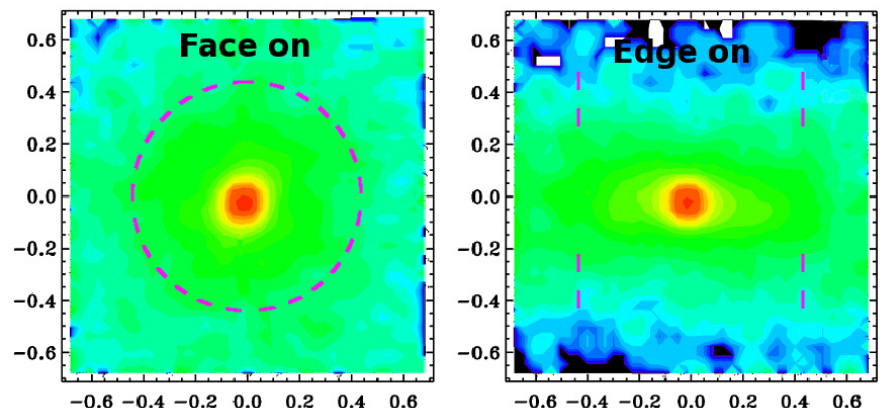

b) M6PsRb: Nested IDs
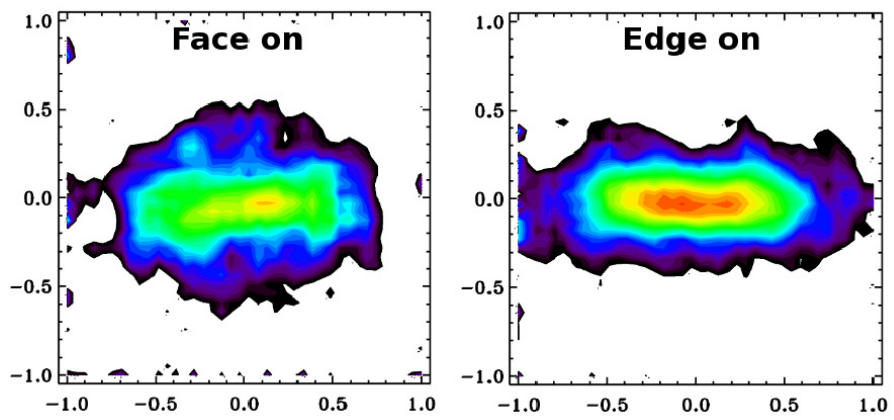

d) M6PIRb: IR embedded in ID
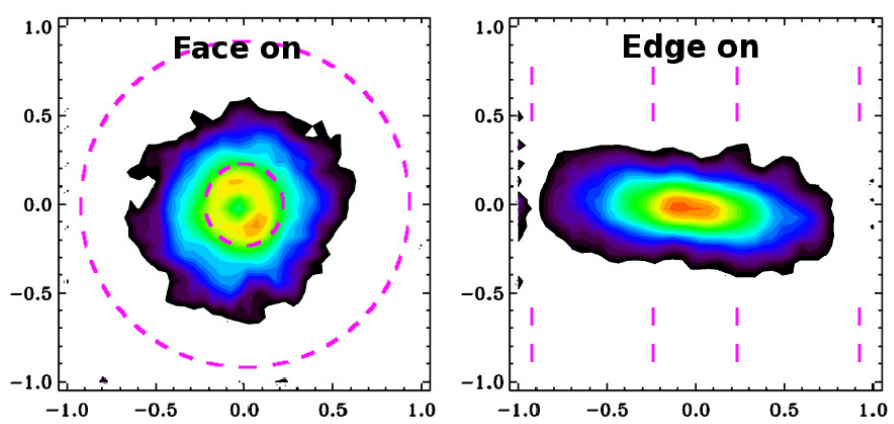

f) M6PsRs: Bulge + ID
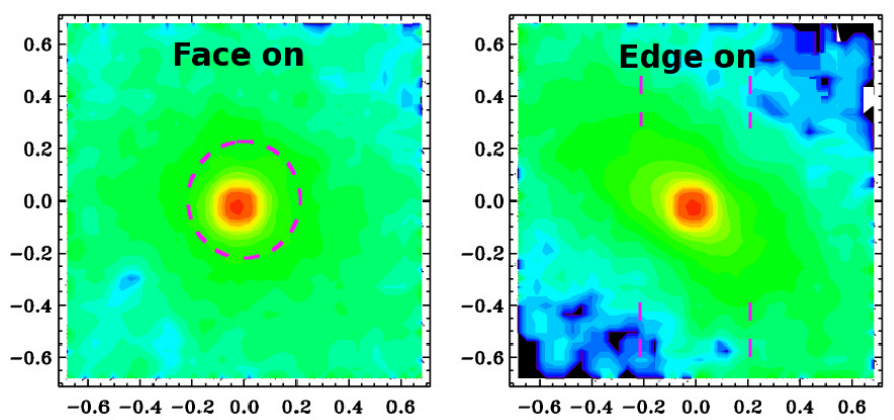

Fig. 4. Morphology of the ICs made out of disrupted satellite stellar material resulting in models with mass ratio 1:6 (models a-f in Table 3 ). Surface density maps of the stars originally from the satellite in the final remnants are presented, using a face-on and an edge-on view (left and right columns in each model, respectively). A rainbow colour palette is used to represent different surface density levels on a logarithmic scale, with redder colours indicating higher values. The levels of the colour scale differ from panel to panel, as they have been set automatically to ensure an adequate sampling of the dynamical range of the surface density shown in each map. Spatial scales in both axes are provided in simulation units. The radial extent of each independent IC (i.e., characterized by an unique exponential profile, see Sect. 3.2.1) are marked with dashed lines in each map.

without experiencing any disruption. Neither of these two models develops a pseudobulge out of disrupted satellite material, because the undisrupted satellite core produces a central peak in the radial surface density profile with a Sérsic index $n \sim 2$ (see the corresponding panels in Fig. 2). Some of the innermost ICs also have a central hole in their radial surface density profiles (see panels d, i, and 1 in Fig. 2). Hence, because of their low thickness, they must be IRs instead of IDs (this is confirmed by surface density maps in Sect. 3.2.3).

The outer ICs are thick in general, following the Sil'Chenko et al. criterion, so we can conclude that these structures basically shape the thick disc in the remnants.

Therefore, the minor mergers produce ICs in the remnant centres out of satellite material that can be described as simple
IDs, nested IDs, IRs hosted by IDs, and bulges hosted by IDs. Although their thickness depends on the case, the central regions of the ICs are generally embedded in the remnant disc and are relatively thin, while the outer ones constitute a thick flared discy structure surrounding the main remnant disc. The global structural characteristics of each IC as derived from their radial and vertical density profiles in Figs. 2, 3 are summarized in Table 4.

\subsubsection{Morphology of ICs}

Figures 4, 5 show the morphology of the ICs formed in each remnant. To provide a 3D description of the IC structure, we plot the face-on and edge-on surface density maps of the disrupted stellar satellite material in all the remnants. The figure shows that the 
g) M9PsDb: Nested IDs

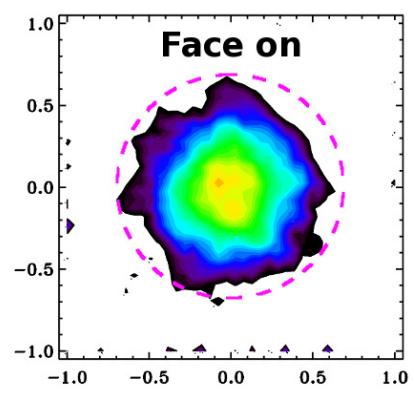

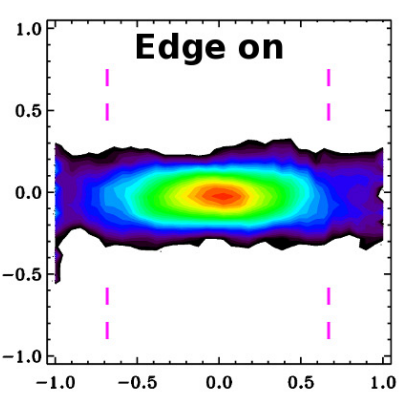

h) M9PsRb: ID

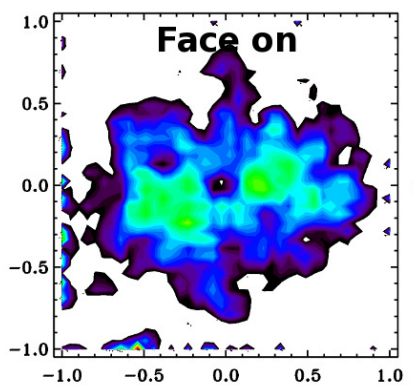

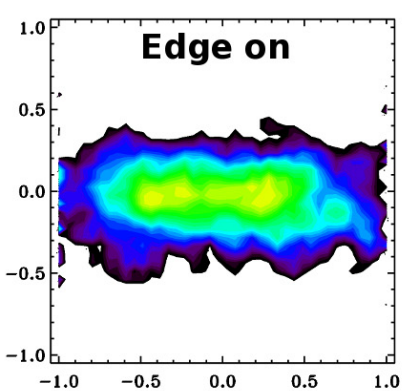

i) M18PsDb: IR embedded in ID
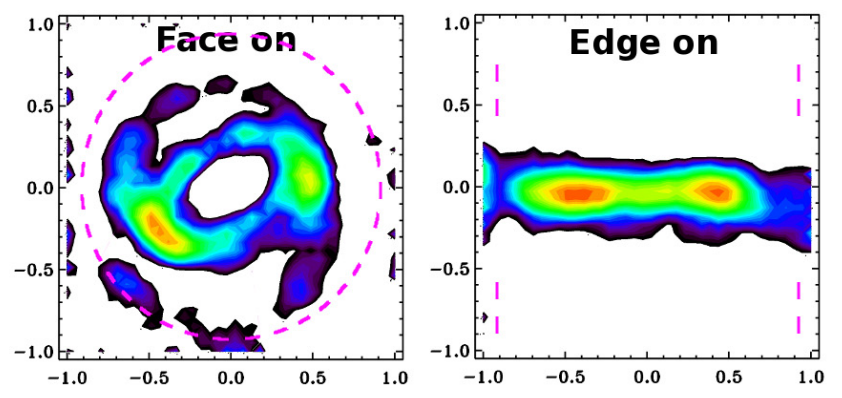

k) M18PIDb: Nested IDs
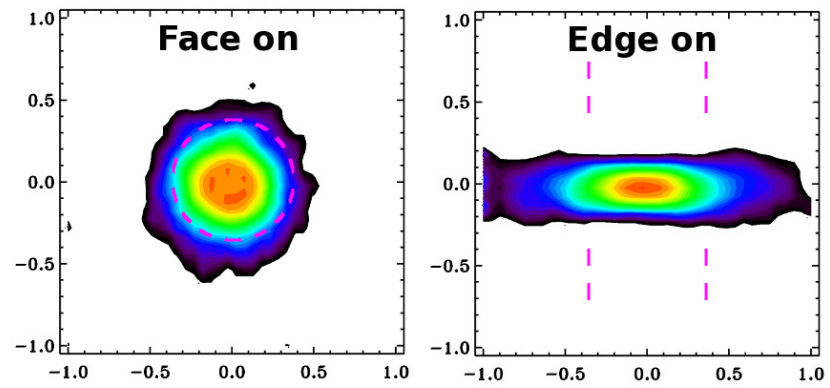
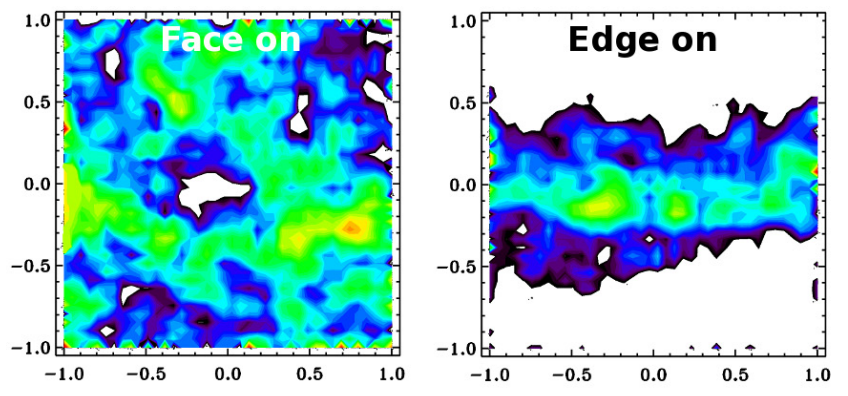

I) M18PIRb: IR embedded in ID

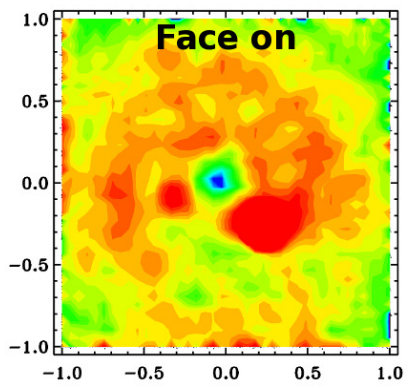

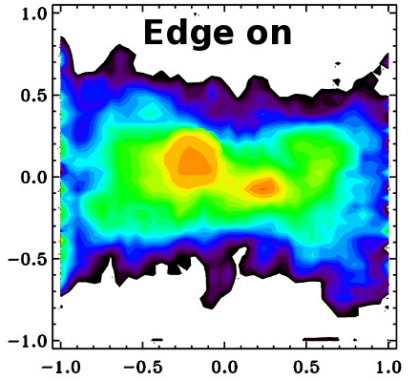

Fig. 5. Morphology of the ICs resulting in models with mass ratio 1:9 and 1:18 out of disrupted satellite stellar material (models g-1 in Table 3). See caption of Fig. 4.

satellite disruption in our experiments has given place to a wide zoo of ICs, all made out of disrupted satellite material, and confirms the global morphology derived from the radial and vertical surface density profiles of Figs. 2, 3 (see Table 4).

Moreover, the maps reveal the existence of substructures than make the morphology of the IC even more complex, such as ring relics (M18PIDb, panel k in Fig. 5), pseudo-rings (i.e., a non-closed ring-like structure similar to the one developed in experiment M18PIRb, panel 1 in Fig. 5), and spiral arms (M18PsDb, panel $\mathrm{i}$ in Fig. 5). The twin clumps observed in some ICs could be tracing weak bars or relics of oval distortions (see panels a, c, and d in Fig. 4 and panels h-i in Fig. 5). The global ellipticity and PA profiles of the final remnants seem to support the oval-related origin of some of these structures (see Sect. 3.3.1). However, none of the remnants has developed a clear nuclear bar in the IC, despite of their having IRs and pseudo-rings. We summarize the substructures found in each case in Col. 3 of Table 4.

Our models demonstrate that minor mergers onto disc galaxies can produce dynamically-cold thin ICs, with varied morphologies ranging from nuclear bars embedded in nested IDs to pseudo-rings, covering all different kinds of inner structures.

\subsubsection{Bars and oval distortions induced by the minor merger}

Numerical simulations have shown that tidal interactions easily produce bars and non-axisymmetric distortions (such as ovals) in thin discs through gravitational torques (see Bournaud et al. 2005a; Mastropietro et al. 2005; Aguerri \& González-García 2009). The time evolution of the density maps of the primary disc material reveals that our simulations are not exceptions: elongated distortions following the directions defined by the orbit of impact are induced in the primary disc by the minor merger (see Fig. 6). Some of these transient bar-like distortions persist until the end of the simulation, despite being considerably weaker than before (as in the case of the figure), but most of them dissolve during the last stages of the remnant relaxation.

The strongest and longest-lived central oval distortions induced by the minor merger in the primary disc appear in the cases with a small primary bulge, corroborating many previous 
Table 4. Characteristics of the ICs formed in the remnants.

\begin{tabular}{|c|c|c|c|c|}
\hline $\begin{array}{l}\text { Model } \\
(1)\end{array}$ & $\begin{array}{c}\text { Global structure } \\
\text { (2) }\end{array}$ & $\begin{array}{c}\text { Substructure } \\
\text { (3) }\end{array}$ & $\begin{array}{l}\text { Rotation } \\
(4)\end{array}$ & $\begin{array}{l}\text { Detectable features } \\
(5)\end{array}$ \\
\hline (a) M6 Ps Db & Nested IDs (thin and thick) & Clumps & Co-rot. & - \\
\hline (b) M6 Ps Rb & Nested IDs (both thick) & Oval distortion & Counter-rot. & $h_{4}>0$ at the ID location. \\
\hline (c) M6 Pl Db & Nested IDs (both thin) & Clumps & Co-rot. & $\epsilon$ and PA trends of weak oval distortion. \\
\hline (d) M6 Pl Rb & IR+ID (thick) & Clumps & Counter-rot. & $\begin{array}{c}\epsilon \text { and PA trends of weak oval distortion. } \\
\text { Ring sections in } h_{3} \text { and } h_{4} \text { maps. } \\
\text { Dumbbell structure in } \sigma \text { map. }\end{array}$ \\
\hline (e) M6 Ps Ds & Bulge + ID (thick) & - & Co-rot. & - \\
\hline (f) M6 Ps Rs & Bulge +ID (thick) & - & Counter-rot. & $\begin{array}{l}\text { Central dip in } \sigma \text { map. } \\
\text { Dumbbell structure in } \sigma \text { map. }\end{array}$ \\
\hline (g) M9 Ps Db & Nested IDs (thin and thick) & Clumps & Co-rot. & $h_{4}>0$ at the ID location. \\
\hline (h) M9 Ps Rb & ID (thin) & Clumps & Counter-rot. & - \\
\hline (i) M18 Ps Db & $\mathrm{IR}+\mathrm{ID}$ (thin) & Clumps, spiral arms & Co-rot. & $h_{4}>0$ at the ID location. \\
\hline (j) M18 Ps Rb & ID (thin) & Irregular clumpy structure & Counter-rot. & Central dip in $\sigma$ map. \\
\hline (k) M18 Pl Db & Nested IDs (thin) & Ring relics & Co-rot. & - \\
\hline (1) M18 Pl Rb & IR+ID (thin) & Pseudo-ring & Counter-rot. & - \\
\hline
\end{tabular}

Notes. Columns: (1) model code; (2) global structure of the formed IC in each remnant, as derived from the radial and vertical surface density profiles shown in Figs. 2, 3 (Sects. 3.2.1-3.2.2); (3) substructure found in the face-on surface density maps of Figs. 4, 5 (Sect. 3.2.3); (4) rotation sense of the IC with respect to the global rotation pattern of the remnant (co- or counter-rotating), as obtained in Sect. 3.2.6; (5) special detectable features present in the global ellipticity and position angle (PA) profiles and in the global kinematical maps of the final remnant, pointing to the existence of these ICs. All the ICs exhibit abrupt changes in the trends of $\epsilon$ and PA profiles at the limits of the ICs, as well as rotation in the centre, so none of these two features are listed in the table (see Figs. 12-15 in Sect. 3.3).

studies that found that a high mass concentration in the galaxy centre tends to stabilize the disc, preventing self-gravity and thus bar formation (see, e.g., Pfenniger \& Norman 1990; Bournaud \& Combes 2002; Athanassoula et al. 2005; Eliche-Moral et al. 2006; Cox et al. 2008). All the transitory bar-like or oval patterns formed in the primary discs rotate in the same direction as the primary disc stars, even in the retrograde mergers.

The final distribution of primary disc material does not show distinct ICs, except in experiment M9PsRb (see Fig. 7), where the primary disc material develops a pseudo-ring at the location of the twin clumps observed in the IC made out of satellite material (see panel h in Fig. 5). Therefore, we are led to conclude that the minor mergers do not drive the formation of IDs or IRs made out of primary material in our experiments, but force the introduction of primary disc material to the centre instead, making the final galaxy bulge grow larger (as reported in EM06).

The resonances that the minor merger induce in the primary disc couple with the satellite disruption, triggering the formation of ICs, such as IDs and IRs (see panels $d$ in Fig. 4, and i in Fig. 5). Nevertheless, we also find that no clear nuclear bars are formed in the IC out of disrupted satellite material. Hence, our models prove that minor mergers can produce IRs and IDs without requiring the development of noticeable bars.

\subsubsection{Alignment of ICs in the remnants}

We analysed the alignment of the different inner structures formed in the merger with respect to the global remnant structure. This analysis is limited in scope because our models explore only two orbit inclinations $\left(30^{\circ}\right.$ and $\left.150^{\circ}\right)$, hence little can be said about the effect of different inclinations on the alignment of the resulting ICs. In Fig. 8, we plot the angles between the angular momenta of the stars originally belonging to the satellite bulge and disc in the final remnant and its total angular momentum (diamonds and triangles, respectively). This figure shows that there is nearly a co-planarity between the IC formed out of satellite bulge material and the final galactic plane of the remnant in all the models (note that their angles are $\sim 0^{\circ}$ in the direct orbits and $\sim 180^{\circ}$ in the retrograde ones). The alignment of the structure formed out of satellite disc material is slightly lower. The near perfect alignment indicates that the plane of the orbit of the satellite has precessed to that of the primary disc by the time the satellite nucleus disrupts, but that precession is incomplete when the satellite loses its disc.

The rotation sense of the IC resulting from the disrupted satellite material can be deduced from this figure: it rotates in the same direction as the main galaxy disc in direct orbits and counter-rotates in the retrograde cases. In general, the alignment between the formed ICs and the main body of the galaxy is worse in retrograde experiments (as already known, see references in Bournaud 2009). Nevertheless, we can conclude that the ICs resulting in these experiments are highly aligned with the final galactic plane in all the cases.

\subsubsection{Rotational support of the ICs}

The ICs formed in our experiments are strongly rotationallysupported, with $V_{\max } / \sigma \sim 2.5$ typically. In Fig. 9, we show the final velocity and velocity dispersion 2D-maps of models M9PsDb and M9PsRb (models $g$ and $\mathrm{h}$ in Table 3), considering all the stars in the remnants, the stars from the primary galaxy, those from the satellite disc, and those from the satellite bulge. The isophotes of the material considered in each panel are also shown, as well as its photometric and kinematic axes. The rotation velocity maps of both models show that the global velocity field of the remnant is governed by the material of the primary galaxy. The existence of a co- (or counter-)rotating IC made out of satellite material in the remnant core negligibly affects the isovelocity contours of the global maps (note that the maps considering all the stars and only those from the primary galaxy in the remnants are extremely similar in both models). Moreover, the existence of these ICs does not appreciably affect the velocity 


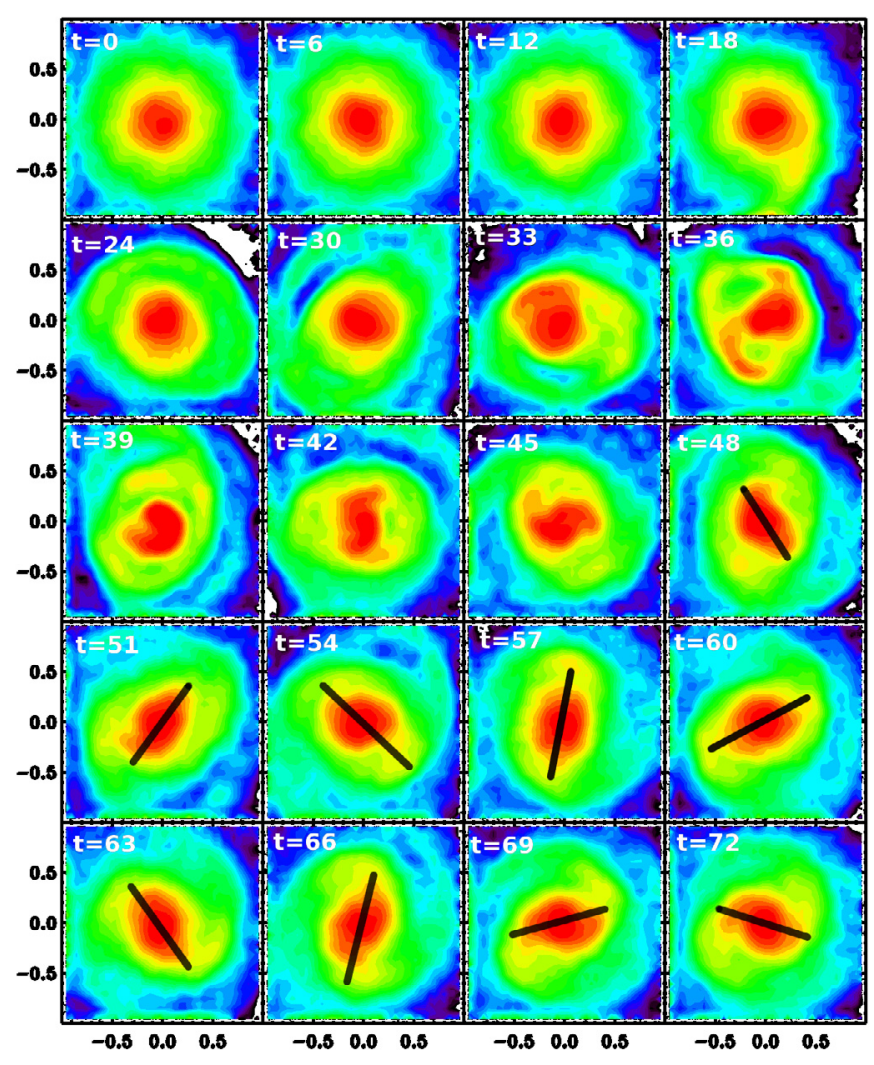

Fig. 6. Formation of a transitory oval distortion in the primary disc material during a minor merger. The time evolution of the surface density map of the primary disc material of model M6PsRs is plotted, using a face-on view of the initial primary galaxy (experiment $f$ in Table 3 ). Time is shown at the top left corner of each panel in simulation units. A rainbow colour palette is used to represent different surface density levels in logarithmic scale, with redder colours indicating higher values. All physical quantities are given in simulation units. The major axis of the oval distortion formed in the remnant disc by the satellite impact is marked with a black line in the last panels. It rotates in the same direction as the primary disc material (clockwise). The orbit and rotation of the accreted satellite is counter-clockwise. The time life of this barlike distortion corresponds to $\sim 0.3 \mathrm{Gyr}$, using the scaling indicated in Sect. 2.

dispersion maps (compare the $\sigma$ maps of each model including and excluding the satellite material).

Nevertheless, the ICs noticeably affect the orientation of the photometric axes with respect to the kinematic ones. As shown in the figure, those obtained by considering just the primary galaxy material are considerably rotated compared to those obtained considering all the stellar content (compare the white axes drawn in the two first velocity maps of the direct model). This is because direct minor mergers drive the formation of large warps in the primary disc (Roškar et al. 2010; Sellwood 2010). The isophotes in the remnant outskirts of the primary disc material trace these warps, rotating the corresponding photometric axes with respect to the orientation (see Fig. 10). However, the addition of a highly-aligned IC in the centre increases the weight of the central regions in the photometry, causing the kinematic and photometric axes to become more similar (compare the kinematic and photometric axes in the first velocity map of the direct model in Fig. 9).

On the other hand, a tight alignment between the photometric and kinematic axes is observed in retrograde mergers, independently of whether we consider the satellite stars in the maps

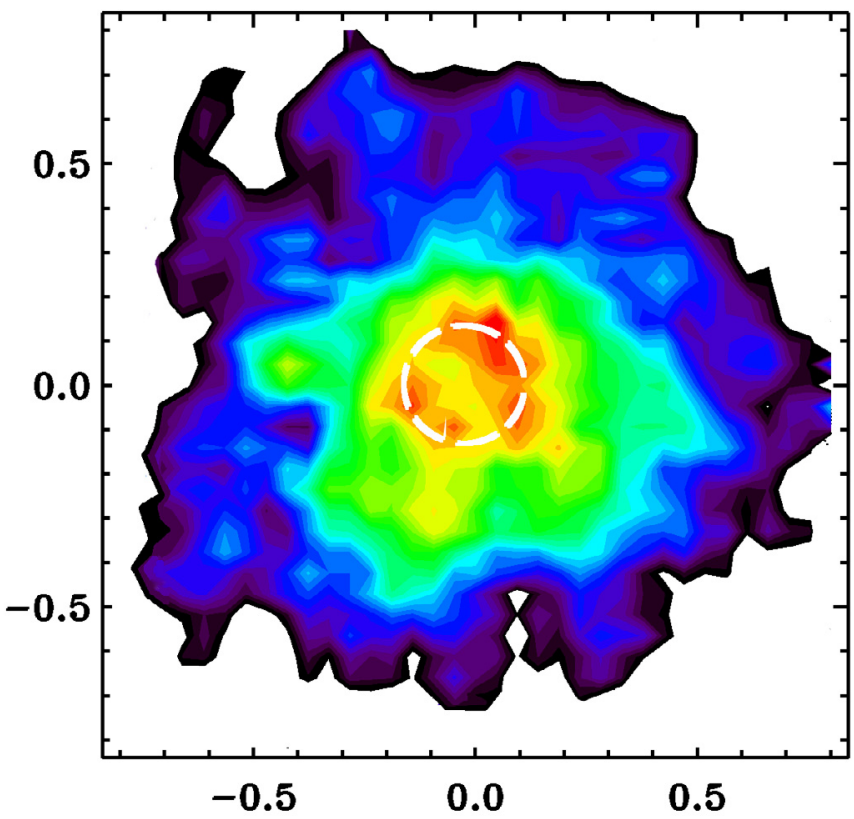

Fig. 7. Pseudo-ring structure in the final remnant of model M9PsRb, made of stars that initially belonged to the primary disc (experiment $h$ in Table 3). It coincides with the location of the two twin symmetric clumps observed in the stellar satellite material in the final remnant. Both substructures are associated with an oval distortion induced by the satellite accretion (see Fig. 5). The pseudo-ring has been marked with a white dashed circle. A rainbow colour palette is used to represent different surface density levels on a logarithmic scale, with redder colours indicating higher values. All physical quantities are given in simulation units.

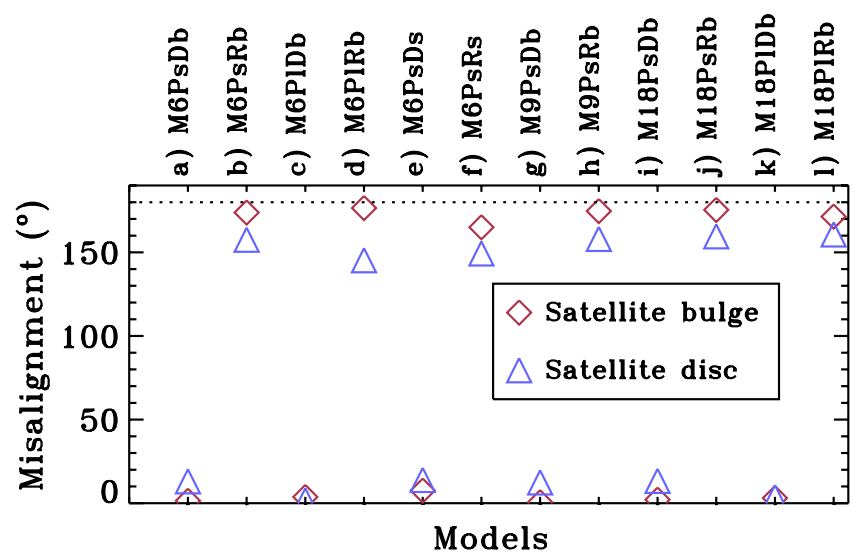

Fig. 8. Misalignment between the material initially belonging to the satellite bulge and disc with respect to the luminous galactic plane of the final remnants (diamonds and triangles, respectively). The letters identifying each model are those used in Table 3.

or not. The reason is that retrograde orbits produce much weaker warps than direct ones because of their lower spin-orbit coupling (compare the velocity maps of the primary disc material in both models of the figure).

The satellite material in the retrograde models counterrotates with respect to the material originally from the primary disc (compare the velocity maps of the retrograde model in Fig. 9). However, no counter-rotation is imprinted by the material coming from the primary galaxy in any model (but see Sect. 3.2.7). Counter-rotating ICs cause a rise in the velocity 

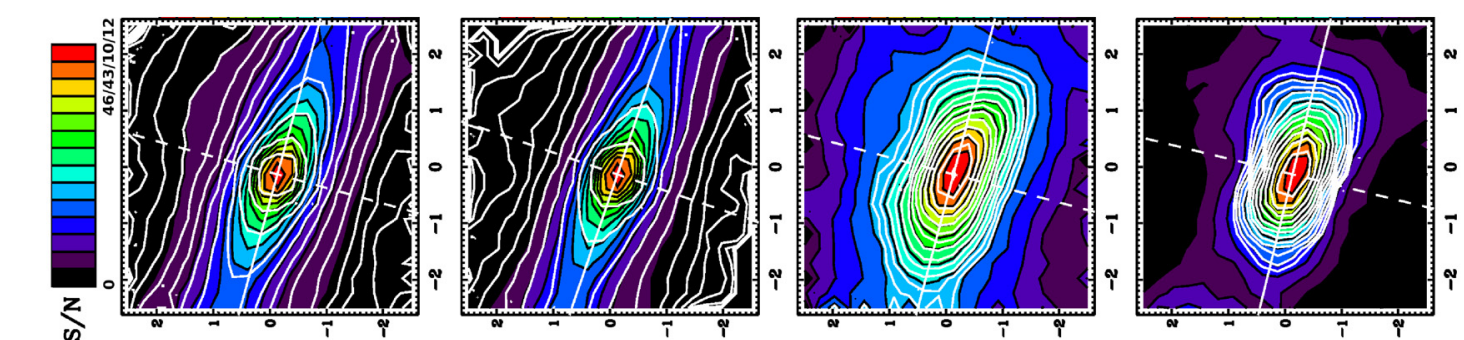

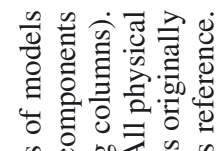
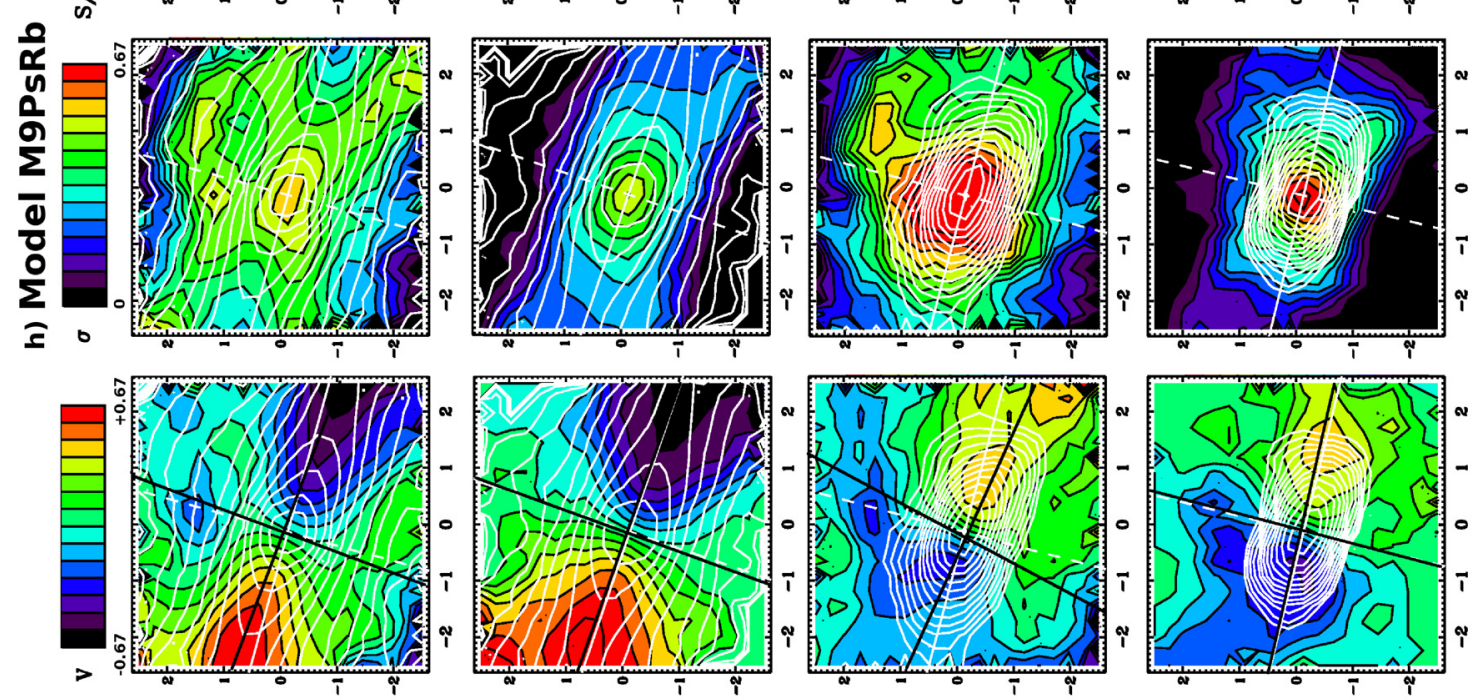

Sנe7s II

Кхереб Клеш!גd
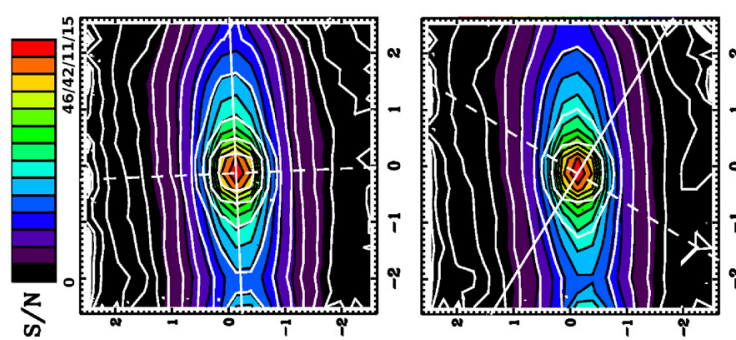

ys!p әң!||әңеs
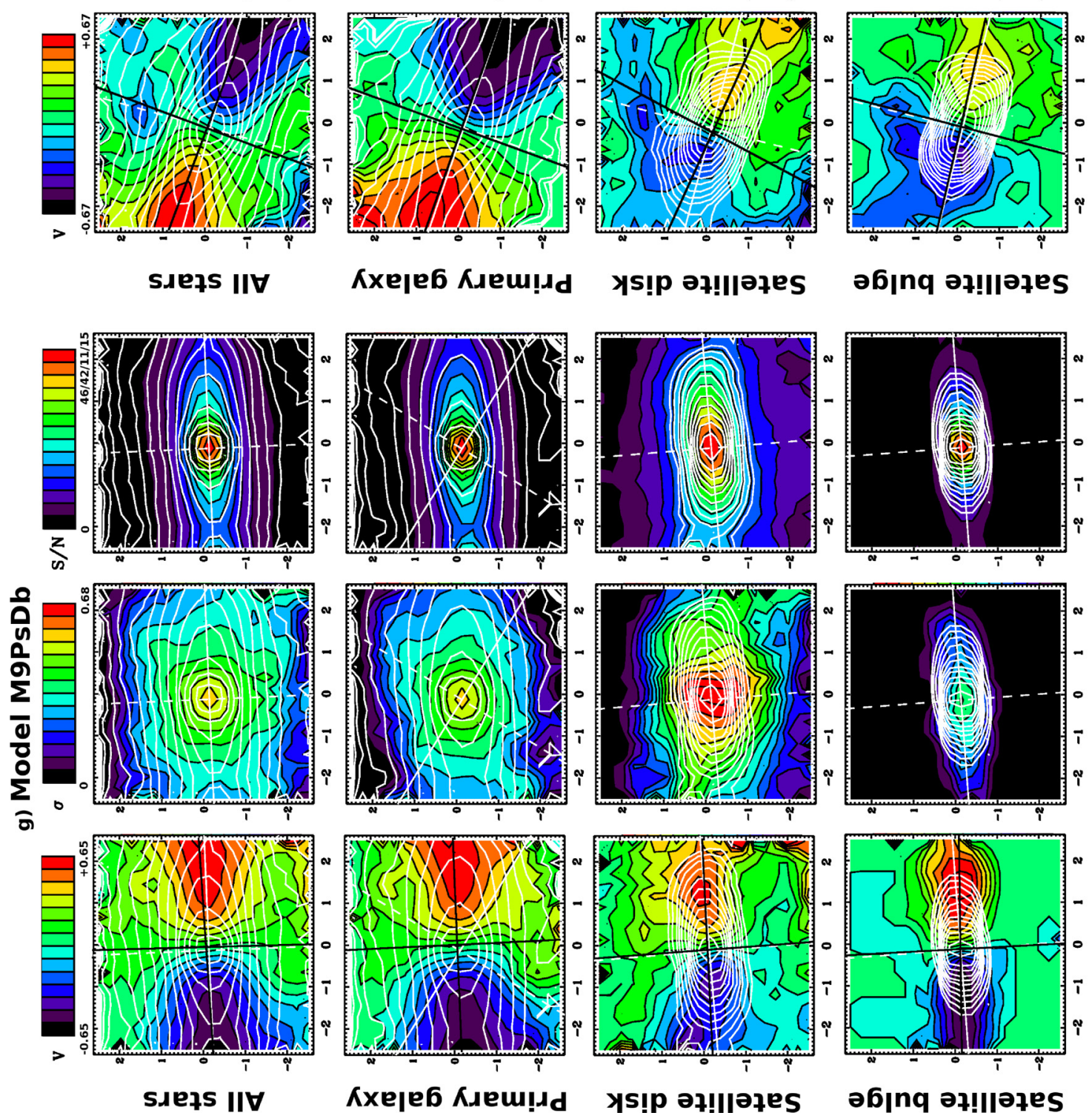

xs!p ә7!||әңеs
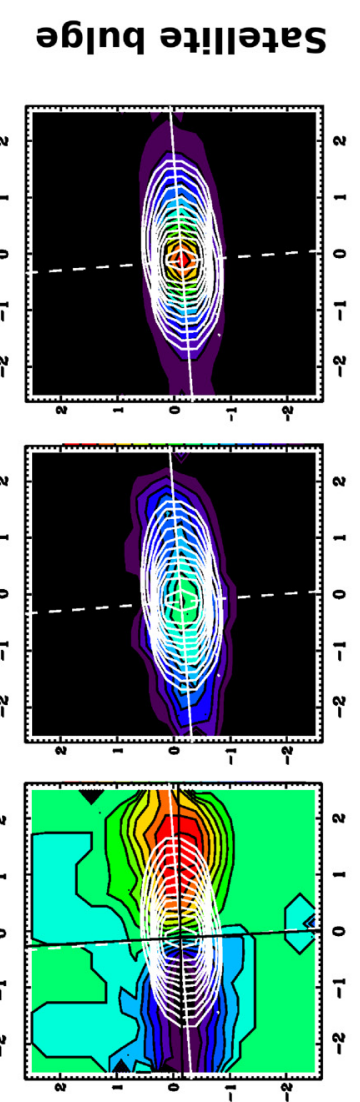

әб|nq әң!||әнеs

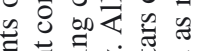

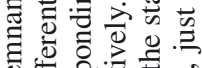

巳

สำ 0

๑

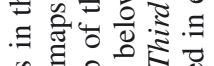

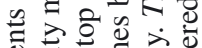

ब.

융

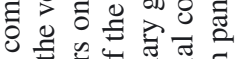

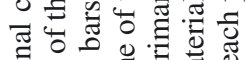

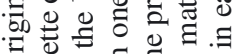

흘

गे

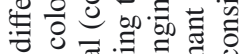

ป. 플 을 0

인

on

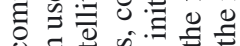

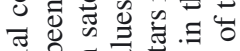

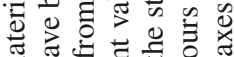

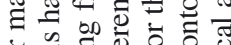

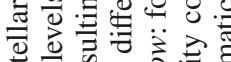

क व 0 ?

ะ

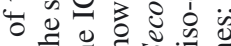

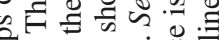

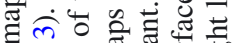

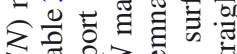

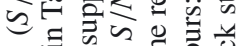

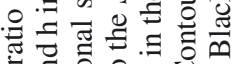

ง

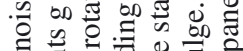

ธิ

$1 . \exists$ :

굴 可

过

กี

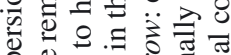

ब.

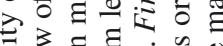

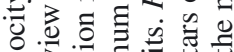

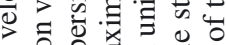

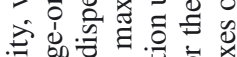

:

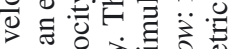

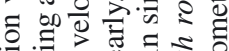

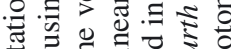

ใิ

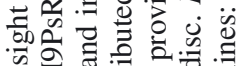

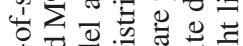

远

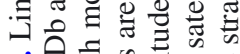

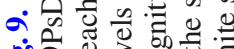

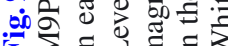




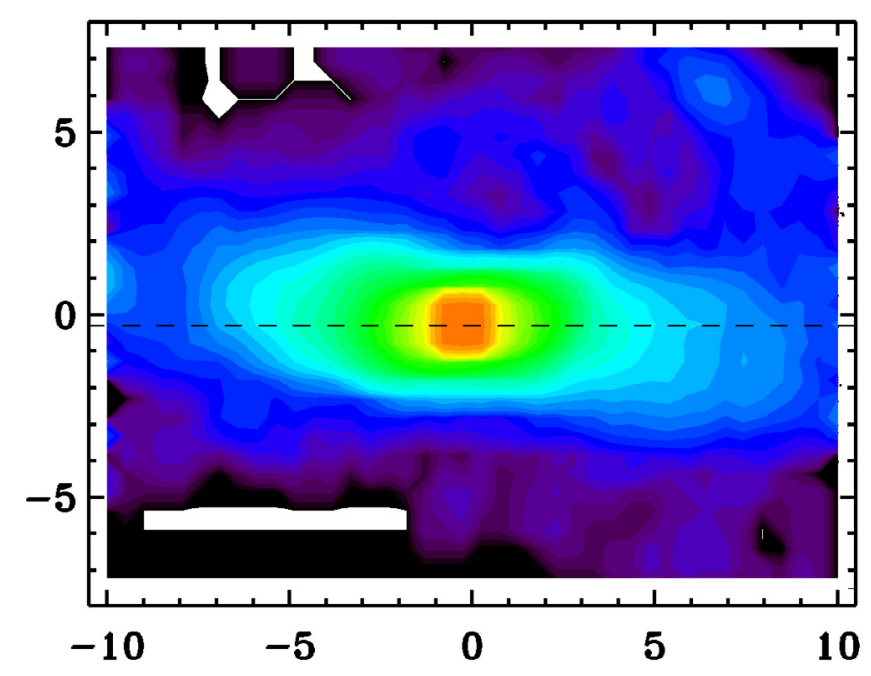

Fig. 10. Final warped disc of model M9PsDb (experiment $\mathrm{g}$ in Table 3). An edge-on view of the material originally belonging to the primary disc in the final remnant of this model is shown. A rainbow colour palette is used to represent different surface density levels with a logarithmic scale, where redder colours indicate higher values. All physical quantities are given in simulation units.

dispersion in the remnant centre, but it does not lead to any significant counter-rotation in the global velocity field of the remnants. Similar conclusions can be derived from analogous maps to the ones shown in Fig. 9 for the other models.

The findings of Sects. 3.2.1-3.2.6 show that minor mergers can produce highly aligned rotationally-supported ICs out of accreted satellite material, without requiring a noticeable redistribution of primary galaxy material through a strong bar or relevant dissipative processes (see Sect. 6). The rotation sense of each IC with respect to the global rotation field of the final remnant for each model is indicated in Col. 4 of Table 4.

\subsubsection{Bender diagrams}

Bender diagrams of our remnants have been obtained using the procedure introduced by González-García et al. (2006). We obtain line-of-sight velocity distributions (LOSVDs) for the merger remnants by choosing a point of view at random for projecting the particle distribution. We then derive a surface density map and define iso-density contours to fit ellipses deriving values for the ellipticity, PA, and the $a_{4}$ parameter (the fourth-order Fourier coefficient measuring the deviation of the iso-density contours from pure ellipses). We place a slit along the major axis of our ellipses out to $R=2$. Given the primary disc scale lengths, our mapping reaches well into the region of the disc. We binned the slit in ten spatial bins and the velocity interval in 50 bins. We derived the radial projected velocity and the number of particles in each bin in velocity for each bin in the slit. In this way we obtain a LOSVD. Finally, we fitted the LOSVD with a Gaussian and the residuals with a Gauss-Hermite polynomial as given by van der Marel \& Franx (1993) and Bender et al. (1994). We repeated this process for each remnant for 90 randomly chosen points of view.

From the fitting procedure, we obtain for each LOSVD a value for the velocity centroid of the distribution at each spatial bin along the slit $(V)$, the velocity dispersion $(\sigma)$, and the amplitude of the third Hermite polynomial $\left(h_{3}\right)$, which is a measurement of the skewness of the distribution.

We show the resulting Bender diagrams for our simulations with 1:6 mass-ratio in Fig. 11 (the results from the other simulations behave similarly). All models present an anticorrelation between the $V / \sigma$ and $h_{3}$ parameter. This is something to be expected because of the prominence of short-axis tube orbits in the progenitor discs that are kept relatively undisturbed owing to the merging event. The large bulge of simulations M6PsDb, M6PsRb, M6PIDb, and M6PIRb (labelled from a to $d$ in Table 3 ) also helps in stabilizing these orbits against the effects of the collisionless minor merger. These simulations differ in terms of either the amplitude of the skewness parameter or the $V / \sigma$ as a consequence of the different orbital parameters of the encounters.

An interesting difference is to be observed in the two plots corresponding to simulations with small bulges in the primary disc: M6PsDs and M6PsRs (models e and $\mathrm{f}$ in Table 3). Here most of the LOSVDs display an anti-correlation between $h_{3}$ and $V / \sigma$ that is typical of discs. However for small values of $V / \sigma$ we find a mild (in the case of M6PsDs) or stronger (in M6PsRs) correlation between the two parameters. This is due to both the effect of the small bulge that is unable to stabilize short-axis tube orbits and to the larger contribution of the satellite bulge in the inner parts of these simulations. It is interesting that the counterrotating orbit of M6PsRs leads to the relatively large correlation signature in the central parts of the diagram.

\subsection{Detection of ICs in the global stellar maps of remnants}

The prominence of the primary stars in the final remnant ensure that the ICs are completely masked by their mass distribution, in such a way that no direct detection of any IC can be performed in either the global surface density maps and profiles of the final remnants. It is encouraging though that indirect detection similar to that attempted and used in observations proves to be successful in many cases, as we show in this section.

\subsubsection{Ellipticity and position angle profiles of the remnants}

Inner structures in real galaxies produce noticeable features in the ellipticity $(\epsilon)$ and PA profiles of the central isophotes of the host galaxies (Erwin et al. 2003; Falcón-Barroso et al. 2004; Chemin \& Hernandez 2009). In the right panels of Figs. 12-15, we have plotted the $\epsilon$ and PA profiles of the stellar material in all the remnants, assuming an inclined viewing angle $\left(\theta=60^{\circ}\right.$, $\phi=20^{\circ}$ ). The radial extent of the ICs defined in Sect. 3.2.1 have been marked in all the plots (vertical dashed lines).

The profiles of the remnants with big primary bulges are dominated by the light distribution of the pre-existing primary bulge in the centre, leading to low ellipticities in the remnant even in the presence of ICs $(\epsilon \leq 0.2$, see the cases shown in Fig. 12, for example). Nevertheless, the existence of ICs (and even their radial extensions) can be deduced by detecting the abrupt changes in the trends of the $\epsilon$ and PA profiles that appear at the transition region between adjacent ICs (for example, between two nested IDs or between an ID and the outer remnant disc, see Figs. 12-15). These trend changes are also observed in real galaxies, where they are associated with isophote twisting produced by bars and triaxial structures in the bulges (see Jungwiert et al. 1997; Erwin et al. 2003).

The existence of weak bar-like or oval distortions in the remnant centre of some models can be deduced from their global isophotal profiles. Models M6P1[D/R]b (panels c in Fig. 12 and $\mathrm{d}$ in Fig. 13) exhibit a slight maximum in $\epsilon$ and a constant PA at the radii where the IC displays twin clumps (see the corresponding panels in Fig. 4). Nevertheless, as mentioned above, none of 

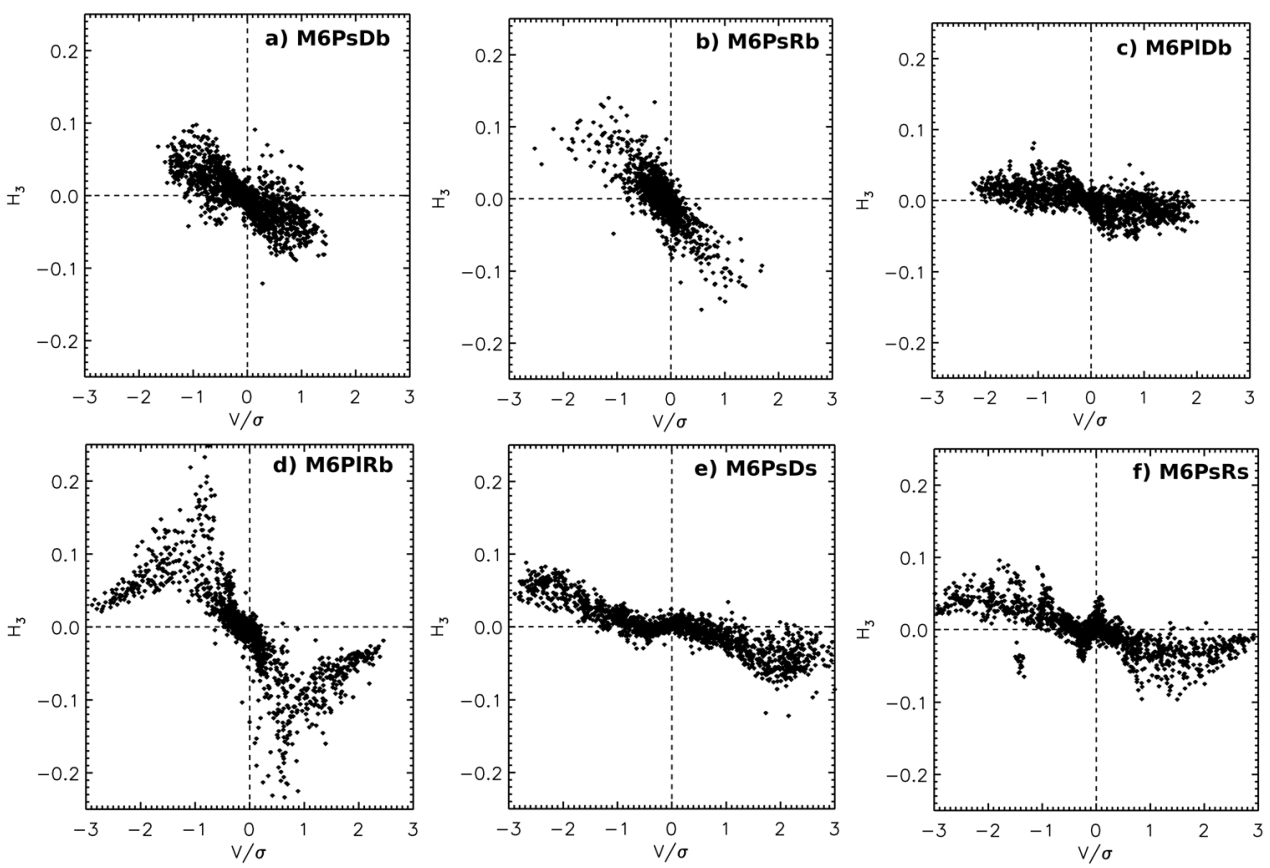

Fig. 11. Bender diagrams of the remnants resulting from the minor merger experiments run with a mass ratio 1:6. The amplitude of the third Hermite polynomial $h_{3}$ of the final remnant is plotted against its value of $V / \sigma$ for 90 randomly chosen points of view.

our remnants develop noticeable nuclear bars, a finding that is consistent with the global ellipticity and PA profiles shown in Figs. 12-15.

The models with a small primary bulge have central ellipticities that are either equal to or greater than that of the outer disc ( $\epsilon \sim 0.3$, see Fig. 13), suggesting that the inner light is dominated by a disc or a flattened triaxial structure (Erwin et al. 2003). The profiles of these models exhibit ellipticity and PA profiles typical of bulge+disc galaxies. The ellipticity displays a rapid rise in the core (bulge) region, a slight decrease in the bulge-disc transition region, and a constant value in the disc outer layers, while the PA is nearly constant outside the bulge region (see Fig. 13). Some particular features in the $\epsilon$ and PA profiles corroborating the existence of some of the ICs identified in Sects. 3.2.1-3.2.3 are listed in Col. 5 of Table 4.

\subsubsection{Kinemetric moments of the velocity fields in the remnants}

In many real galaxies, the existence of kinematically-decoupled components that are not detectable through direct imaging is inferred from special features in their kinematic maps. In most cases, the existence of these ICs is later confirmed by unsharp masking or direct detection of its gas component in [OIII] or $\mathrm{H} \beta$ emission-line maps (see, e.g., Falcón-Barroso et al. 2003, F06).

Figures 12-15 show the 2D-maps of the kinemetric moments of the line-of-sight velocity distribution of all the luminous material in each remnant, using edge-on views. The line-of-sight velocity $V_{\mathrm{LOS}}$, the velocity dispersion $\sigma$, and the third- and fourth-order coefficients of the Gauss-Hermite series $h_{3}$ and $h_{4}$ are shown for each model. They were obtained by adapting the profit routine (originally designed for profile fitting of spectral emission-lines by Gauss-Hermite series) to $N$-body data (Riffel 2010). The rotation velocity maps in these figures differ from the analogous ones presented in Fig. 9 in terms of the spatial resolution. While those presented in Fig. 9 use a uniform spatial binning, the ones shown in Figs. 12-15 have higher spatial resolution in the centre than in the outskirts to improve the $S / N$ of the estimates of the kinemetric moments in low-density regions (analogously to what is done for observational data, see F06). The central spatial resolutions in these figures are similar to those achieved by current observations in the nearby Universe, adopting the scalings proposed in Sect. $2(\sim 0.5 \mathrm{kpc})$. Regions with low $S / N$ have been masked in the maps.

Figures 12-15 show that the existence of many ICs could be deduced from these maps of kinemetric moments. Many particular features present in these maps that corroborate the existence of some of the ICs identified in Sects. 3.2.1-3.2.3 are indicated in Col. 5 of Table 4. However, we must note that these features are smooth as all the maps of the kinemetric moments show large dispersion (specially, at outer radii), hence they are detectable only in case of low noise levels. Some of these kinematical features include:

i. Misalignment of kinematic axes. A clear misalignment of the inner and outer kinematic axes of the galaxy is detected in some cases (see model M6PsRb, panel b in Fig. 12), which can be produced by noticeable disc warping (as shown in Fig. 10). Significant rotation extends to the central regions in all the remnants. The final rotation fields, although following a typical spider-like diagram, seem quite distorted in general, as is characteristic of dissipationless models (Jesseit et al. 2007). In general, experiments with lower mass ratios or larger pericentres imply that there has been a smoother destruction of the initial galaxy rotation pattern.

ii. Stretching of iso-velocity contours in the centre. The existence of co-rotating IDs and IRs leads to larger measurement of rotation velocity at the centre, stretching the isovelocity contours towards the major axis at their location. This causes the angle of these contours to become more open at the remnant outskirts than in the centre (see, e.g., models M6PsDs and M6PIDb, panels b and c in Fig. 12).

iii. Twisting of central iso-velocity contours in retrograde models. As shown in Sect. 3.2.6, retrograde models contain counter-rotating ICs. In some of these models, the 
contribution of this IC to the mass in the remnant core is high enough to imprint a strong twisting of the iso-velocity contours by $\sim 90^{\circ}$ at the core region (see model M6PsRb in panel b of Fig. 12, and models M18P[s/1]Rb in panels $\mathrm{j}$ and 1 of Fig. 15).

iv. S-shaped twists of iso-velocity contours. Almost all the cases exhibit S-shaped or integral-sign-shaped twisting of the central iso-velocity contours at a certain height in the galactic plane. These features are produced by the sharp decrease in the amount of stars that contributes to the velocity field at a certain spatial position. Therefore, S-shaped kinematic twists in our models appear at the spatial locations where the mass contribution of the ICs becomes negligible (see, e.g., models M6PIRb and M18PsDb, in panels d and i of Figs. 13 and 14).

v. $\quad h_{4}$ peaks. The majority of the models with big primary bulges, in general, have positive $h_{4}$ values peaking at the location of the ICs formed at the end of the simulation. In particular, the ring geometry of the IC resulting in model M6PIRb can be deduced from the two well-defined symmetric peaks present in the $h_{4}$ maps, which trace its perpendicular sections (panel d in Fig. 13).

vi. Correlations between the kinemetric moments. We have found that $v_{\mathrm{LOS}}$ and $h_{3}$ correlate at the location of the formed IC in our remnants if it exhibits oval or barlike distortions (see models M6Ps[D/R]b and M6PIDb in panels a-c of Fig. 12, and models M9PsRb and M18PsDb in panels h-i of Fig. 14). However, when the ICs show no signs of these distortions, $v_{\mathrm{LOS}}-h_{3}$ anti-correlate at the location of the IC, accordingly to the discy structure of the majority of the obtained ICs (see models M18PsRb and M18Pl[D/R]b in Fig. 15). Some models also exhibit a mixed behaviour: $v_{\mathrm{LOS}}-h_{3}$ correlate at the remnant core (at $R<0.3$ ) and anti-correlate at the position of the $h_{4}$ peaks, as occurs in models M6PsDs (panel e in Fig. 13) and M9PsDb (panel $\mathrm{g}$ in Fig. 14). This is probably indicative of an origin related to oval distortions and bar-like distortions in these components.

vii. Dumbbell $\sigma$ structures. Dumbbell structures in $\sigma$ maps point to the existence of counter-rotating IDs, where the $\sigma$ tends to rise at their location. Although all the retrograde models correspond to counter-rotating IDs or IRs, dumbbell structures are only observed in models M6PIRb and M6PsRs (panels $d$ and $f$ in Fig. 13).

viii. Central $\sigma$ dips. In general, the velocity dispersion is very large at the remnant centres because the bulge dynamics dominate at these radii. However, it tends to decrease at the bulge-to-disc transition region because of the contribution of the co-rotating IC. In our models, $\sigma$ dips are detected only in the models of small primary bulges (see panels e and $f$ of Fig. 13).

To summarize, the velocity fields of our remnants exhibit features that reveal the existence of the central ICs formed through the satellite accretion, such as the stretching of the iso-velocity contours in the centre, S-shaped kinematic twists, and dumbbell $\sigma$ profiles. More inclined views of the remnant smooth out these features, making the ICs undetectable in the velocity 2Dmaps.

\section{Qualitative comparison to observations}

We now qualitatively compare the properties of the ICs obtained in our minor merger simulations with real observational cases, to emphasize their similarities and differences.

\subsection{Structural comparison}

Figure 16 compares the morphology of some of the ICs formed in our experiments to observational examples that exhibit similar inner structures, from the sample of Erwin \& Sparke (2003). As shown by the figure, the ICs resulting from our minor merger experiments are analogous structural and morphologically to the ICs hosted by many real spiral galaxies. The IDs, nested IDs, rings and pseudo-rings, spiral patterns, and even undisrupted clumps with irregular spatial distributions as those observed in our experiments are all found in real spiral galaxies (see also Buta \& Combes 1996; Erwin \& Sparke 2002; Erwin et al. 2003; Erwin 2004; Pérez-Gallego et al. 2010; Sil'Chenko et al. 2011).

Star-forming pseudo-rings are substructures that are usually associated with gas and starbursts in real galaxies (Shapiro et al. 2010; Sil'Chenko 2010; F06). However, although our models do not consider dissipative components, some remnants exhibit stellar ring relics or pseudo-rings in the centres (see models $\mathrm{M} 18 \mathrm{Pl}[\mathrm{D} / \mathrm{R}] \mathrm{b}$ in panels $\mathrm{k}$ and $\mathrm{l}$ of Fig. 5).

Our remnants have not developed significant nuclear bars, although some so display weak ovals or bar-like distortions at their centres (see Sect. 3.3.1). The IRs and pseudo-rings that appear in some of our models are then not directly associated with well-formed bars. They have instead formed by the coupling of the satellite disruption to the resonances induced in the primary disc by the encounter. Cases of IRs and sets of concentric rings not associated with bars are also found in real galaxies (Buta \& Combes 1996; Muñoz-Tuñón et al. 2004; Kormendy et al. 2006). In addition, our models point to the resonance-related origin of IRs, independently of whether these resonances are linked to either strong bars or weak non-axisymmetric perturbations as in the ovals found in some of our models (see Sect. 3.2.4).

The stellar IDs formed in our experiments have radial lengths ranging from $\sim 0.4$ to $\sim 1$ disc scale-lengths of the final remnant, which are equivalent to radial lengths spanning from $\sim 360 \mathrm{pc}$ to $\sim 2.5 \mathrm{kpc}$, adopting the scaling provided in Sect. 2 . These values are in excellent agreement with those obtained for the IDs detected in real galaxies (Erwin \& Sparke 2002; Sarzi et al. 2006; Shapiro et al. 2010; Sil'Chenko et al. 2011, F06). The IRs and pseudo-rings formed in our simulations are embedded in these IDs, thus typically have smaller linear sizes. Adopting the scalings of Sect. 2, our IRs closely match the standard 1 kpc-radius IRs that lie between the inner Lindblad resonances of many disc galaxies (Erwin \& Sparke 2002; Shapiro et al. 2010).

The flared vertical structure of most nested IDs produced by our models (see Fig. 3) is extremely similar to the one derived for the galaxy NCG 7217, which consists of two large-scale nested stellar discs (Sil'Chenko et al. 2011).

The existence and extension of many real ICs are usually inferred from abrupt changes in the trends of the ellipticity and PA profiles of the global galaxy isophotes (Erwin \& Sparke 2002, 2003; Erwin 2004). As we have shown in Sect. 3.3.1, all the ICs formed in our models display similar features in the global profiles of the final remnants, in agreement with observations (Prieto et al. 2001; Erwin \& Sparke 2003).

The small misalignment between the ICs obtained in the simulations and the global galaxy plane (see Sect. 3.2.5) is in excellent agreement with observations: F06 report misalignments 
of typically $\lesssim 20^{\circ}$ for the ICs harboured by $\mathrm{Sa}-\mathrm{Sb}$ galaxies, and Krajnovic et al. (2011) find that $90 \%$ of the ICs in a sample of 260 early-type galaxies can be considered to be aligned to better than $5 \mathrm{deg}$. Nevertheless, we cannot discard the possibility that this result may be facilitated by the moderate inclinations of our simulated orbits. The rapid decay of the satellite orbit to the primary disc plane observed in all the models suggests that more inclined orbital configurations could similarly result in aligned ICs, and that extreme orbital inclinations would be required to produce noticeably misaligned ICs.

Our simulations also demonstrate the feasibility of the scenario proposed by F06 to explain the small misalignment of the ICs observed in Sa-Sb galaxies compared to those in E-S0's (usually above $60^{\circ}$, see Sarzi et al. 2006). According to these authors, if the ICs resulted from minor mergers, the morphology of the primary galaxy in the encounter should play a crucial role in determining the IC alignment. In our simulations, the evolution of the satellite orbit to the primary disc plane prior to its disruption is produced by the low spheroidality of the primary galaxy potential, which establishes a privileged plane a priori (the primary disc plane). Spheroidal potentials (such as those of E-S0 galaxies) do not have any preferential axis of symmetry, thus they do not produce this effect in the orbits of the accreted satellites (Di Matteo et al. 2008). Therefore, our simulations suggest that, if the majority of ICs originated from minor mergers, we should detect more aligned ICs in disc galaxies (as Sa-Sb's) than in spheroidal ones (E-S0's), in agreement with observations and the arguments of F06. Moreover, F06 propose that the large amounts of gas involved in the merger must contribute to this alignment. We note that, although the previous sentence must be true, our models prove that gas is not strictly necessary to obtain high co-planar ICs in minor mergers.

\subsection{Kinematical comparison}

ICs disturb the velocity field of their host galaxies, producing particular kinematical features in the global maps of the galaxy. Our remnants exhibit many of these features, such as disturbed iso-velocity contours in the centre, significant $\mathrm{S}$-shaped kinematic twists at the limiting radii of the ICs, $\sigma$ peaks, dumbbelllike $\sigma$ structures associated with counter-rotating IDs, and the stretching of iso-velocity contours in the case of co-rotating ICs (see Sect. 3.2.6), which are all similar to those observed in real galaxies harbouring ICs (see F06; Sarzi et al. 2006; Krajnović et al. 2008).

The counter-rotating ICs formed in our models are so embedded in the primary disc stellar material that, in general, they do not imprint noticeable counter-rotation at the centre of the remnant (Sect. 3.3.2). The masses of the ICs are too low to significantly affect the central dynamics of the remnants, which are basically dominated instead by the host rotating disc (see Sect. 3.2.6), although some counter-rotation becomes detectable at specific viewing angles (see model M6PsRs in panel $\mathrm{f}$ of Fig. 11). Therefore, the presence of large disc components in galaxies makes the observational detection of counter-rotating ICs difficult. This can explain why the observational samples of early-type spirals exhibit an apparent absence of counter-rotating features relative to the samples of E-S0's, which lack the relevant discs (see Kannappan \& Fabricant 2001; F06). The frequency of counter-rotating ICs in both E-S0's and Sa-Sb's could be similar, the discs of Sa-Sb's being responsible for masking them.

Our collisionless models reproduce several trends and correlations between the kinemetric moments of real galaxies with ICs, although they also display several differences. In general, our models show $h_{4} \geq 0$ and $h_{4}$ peaks at the location of the ICS, in excellent agreement with observations of ICs in early-type galaxies (F06; Krajnović et al. 2008). However, the $V_{\mathrm{LOS}}-h_{3}$ correlation found in our simulations partially reproduce the observational trends. Observations report a $V_{\mathrm{LOS}}-h_{3}$ anti-correlation at the location of co-rotating ICs in Sa-Sb's, and a correlation if the ICs counter-rotate (F06). Nevertheless, our models tend to predict a $V_{\mathrm{LOS}}-h_{3}$ anti-correlation, independently of the rotation sense of the IC relative to the final galaxy (see Figs. 12-15). Although some retrograde models can display correlations for particular viewing angles (see model M6PsRs, panel $\mathrm{f}$ in Fig. 11), in general we find anti-correlations (see the remaining Bender diagrams in Fig. 11).

Our models do not account for the effects of gas dynamics and star formation, which can obviously affect the kinematical structure of the final remnant (see Sect. 5). Collisionless remnants are known to contain box orbits, which cause $V_{\text {LOS }}$ and $h_{3}$ to become correlated (see, e.g., Naab \& Burkert 2001). However, the inclusion of gas in the simulation has the opposite effect: it suppresses the formation of box orbits and causes $V_{\mathrm{LOS}}$ and $h_{3}$ to be anti-correlated at the remnant centre (Bendo \& Barnes 2000; Naab \& Burkert 2001; Jesseit et al. 2007). Our collisionless simulations are then atypical in this sense, as they tend to exhibit $V_{\mathrm{LOS}}-h_{3}$ anti-correlations. This trend was already obtained by González-García et al. (2006), who showed that these anticorrelations can be maintained in a collisionless merger simulation whenever a central bulge allows the discs to retain some of their original angular momentum during the merger, leading to short-axis tube orbits still being present in the final remnant.

In our models, a $V_{\mathrm{LOS}}-h_{3}$ correlation seems to be more closely related to the existence of bar-like or oval distortions than to counter-rotation (Sect. 3.3.2). The properties of our remnants can be reconciled with the observational trends if we consider that retrograde mergers produce longer-lasting bar-like instabilities (although weaker) than their direct analogues (Barnes \& Hernquist 1996). Therefore, we should expect statistically to find more bar-like distortions and ovals in the ICs resulting from retrograde mergers than in those coming from a direct encounter. Assuming that a $V_{\mathrm{LOS}}-h_{3}$ correlation is related to the existence of non-axisymmetric distortions in the galaxy disc, the previous result establishes a correspondence between $V_{\text {LOS }}-h_{3}$ correlations and counter-rotating ICs, in agreement with the results of F06. This scenario is consistent with the different $V_{\mathrm{LOS}}-h_{3}$ trends displayed by Sa-Sb and E-S0 galaxies. While ICs hosted by early type galaxies are always associated with $V_{\mathrm{LOS}}-h_{3}$ anticorrelations (Bender et al. 1994), the ICs in Sa-Sb's exhibit correlations only if the IC is counter-rotating (F06). This can be explained if E-S0's more efficiently inhibit the formation of bars and ovals than Sa-Sb's (because they have larger bulges and negligible disc components), which should then in turn explain why the ICs in these galaxies exhibit $V_{\mathrm{LOS}}-h_{3}$ anti-correlations in general (in agreement with observations).

In conclusion, minor mergers could account for the existence of many stellar dynamically-cold ICs in spiral galaxies and even in E-S0's. The present models demonstrate that they can produce ICs with geometrical, structural, and kinematical properties similar to those observed in real galaxies.

\section{Model limitations}

We have analysed the role of minor mergers in the formation of dynamically-cold thin stellar ICs, without accounting for gas and star formation effects. Obviously, the inclusion of dissipative components in the models would not just provide the 
subsequently formed IC with an additional recent stellar population or gas component, but could also noticeably affect its final structure and kinematics.

The formation of IDs and IRs are associated with the redistribution of angular momentum in the remnant disc. In this sense, gas components are expected to contribute noticeably to this re-distribution, mainly during disc distortions. Nevertheless, some studies have demonstrated that dissipative components (although relevant) are neither essential nor decisive in the formation of kinematically-decoupled ICs through major mergers. Gas causes the remnants to appear more round and axisymmetric, wiping out small kinematical misalignments more easily, but the resulting IC is structurally similar to the one formed in the collisionless analog (Jesseit et al. 2007).

Although star formation triggers the formation of young stars within the IC in simulations of major mergers, the resulting IC still consists of a relevant old stellar component (Di Matteo et al. 2008). If the formation of an ID or IR depended basically on merger-induced gas inflows to the galaxy centre and on the subsequent star formation (Barnes \& Hernquist 1996; Bournaud et al. 2005b), these substructures should be bluer or, at least, younger than the surrounding bulge component. However, this is not the case, as IDs usually exhibit very similar colours to those of their host bulges (see Morelli et al. 2004; Peletier et al. 2007). Therefore, although gas and star formation effects must have been relevant to the formation of IDs and IRs, they might not be essential in many cases. This view is supported by the significantly old underlying stellar component detected in most IDs and IRs (Buta \& Purcell 1998; Buta et al. 1998; van den Bosch et al. 1998; Krajnović \& Jaffe 2004; Morelli et al. 2004).

The flattened structure of IDs and IRs has been traditionally interpreted as a sign of the essential role played by gas in their formation (Cappellari et al. 2007), but our models prove that central thin rotationally-supported ICs can result from satellite disruption without supplying gas to the remnant centre. However, this is a simplified picture of the reality, as the majority of the observed IDs and IRs contain recent (or ongoing) star formation, dust, and gas, which clearly points to the tight relation between dissipative processes and their buildup (see, e.g., Barnes \& Hernquist 1996; van den Bosch \& Emsellem 1998; Morelli et al. 2004; Kormendy et al. 2005; Cappellari et al. 2007; Peletier et al. 2007, F06). We expect that the inclusion of gas and star formation in the models would have made the ICs formed more detectable, accounting for the star formation that minor mergers are usually found to trigger in the centre of galaxy discs (Kaviraj et al. 2009). Hence, we intend to re-run these simulations including gas and star formation processes in the near future, to determine the effects of dissipative processes on the formation of ICs.

\section{Discussion}

The present models provide novel perspectives of the buildup of dynamically-cold ICs through minor mergers, because the resulting IDs and IRs do not result from either primary disc material or a gaseous component as in other studies, but from the disrupted stellar satellite material. Traditionally, minor mergers have been considered as secondary drivers of the formation of IDs or IRs in galaxies, in the sense that the rotationallysupported ICs were thought to come from the bars triggered in the discs by the encounters, not by the minor merger themselves. The accreted satellite material did not help to form the ICs, except when it reached the centre undisrupted (see Sect. 1). Therefore, minor mergers have been considered just as the agents inducing bars, but the bars were the processes responsible for the buildup of the ICs.

Our models demonstrate that both processes (the induced disc resonances and the accretion of material external to the galaxy) are closely connected, as the resulting IC is made out of satellite material and clearly originates in transitory nonaxisymmetric distortions of the galaxy disc (Sects. 3.1-3.2). The difference between our models and previous ones from the literature is basically the satellite characteristics, as we have found that very different orbital configurations can produce similar ICs (see Table 4). In our models, the discy structure and the realistic density contrast of the satellites with respect to the primary galaxies ensure that they are quite likely to be disrupted, yet resistant enough to reach the remnant centre (in contrast to previous models, see Aguerri et al. 2001; Moster et al. 2010). This implies that, if a low-density satellite (as a dS) were accreted by a galaxy, the existence of a prominent bulge in the primary galaxy would induce the complete disruption of the satellite and the formation of an IC out of it. However, if the accreted satellite were dense (such as a dSph or $\mathrm{dE}$ ) or if the primary galaxy had a small bulge, the undisrupted satellite core would be deposited at the centre without being disrupted, increasing the spatial density at the galaxy core.

The bar-related origin of numerous bulges and dynamicallycold ICs in galaxies is indisputable (Martinez-Valpuesta et al. 2006; Berentzen et al. 2007; Romero-Gómez et al. 2007; Athanassoula et al. 2009a,b, 2010; Bagley et al. 2009; Fisher \& Drory 2010), but it is also true that more than one third of these structures in Sa-Sb galaxies appear to be unrelated to bars (see references in Sect. 1). Considering the relevance that minor mergers seem to have had to galaxy evolution (see Jogee et al. 2009; López-Sanjuan et al. 2010a,b), it is probable that many bars may even have been induced by minor mergers. Hence, the present models provide a feasible explanation of old, pure stellar IDs and IRs in unbarred galaxies (especially of the counterrotating cases, see Jesseit et al. 2007; Morelli et al. 2010).

The mixing of material observed in the models (owing to the merger and/or to the triggered oval distortions) indicates that it would be very difficult to prove the minor merger origin of the resulting ICs by disentangling the stellar population with an external origin from the underlying stars. However, the minor merger origin of the ICs in some galaxies is obvious, mainly if the IC is counter-rotating or it is harboured by a non-relaxed host (see Haynes et al. 2000; Gutiérrez et al. 2002; Shapiro et al. 2010; Sil'Chenko et al. 2011).

The key role of minor mergers in the growth of the bulges of spiral galaxies is clearly evident, because the relics of disrupted satellites and ongoing minor mergers are frequently found in nearby galaxies, indicating that these processes are extremely common (Martínez-Delgado et al. 2007, 2008, 2009, 2010; Knierman 2010). Therefore, the present models suggest that the majority of the ICs found in spiral galaxies might have had a minor-merger related origin (independently of whether the minor merger triggers a noticeable bar or not), and that the role of minor mergers in the formation of ICs may have been much more complex than leading to the production of a bar, as traditionally assumed.

\section{Summary and conclusions}

We have investigated the capability of minor mergers to trigger the formation of IDs and IRs in spiral galaxies, using collisionless $N$-body simulations. We have extended the simulations of the minor mergers of disc galaxies presented in EM06, sampling a wider parameter space of initial conditions. Different orbits 
and mass ratios have been considered, as well as two different models for the primary disc galaxy (Sab or Sc galaxy).

All the simulated minor mergers have developed thin rotationally-supported ICs out of disrupted stellar satellite material, with scale-lengths analogous to those observed in real IDs and IRs. The resulting ICs are closely aligned with the main galactic plane of the remnant, as the original non-spheroidal potential of the primary galaxy causes the satellite orbit to evolve to its privileged plane prior to disruption. This provides a possible explanation of the small misalignment observed in the ICs found in Sa-Sb galaxies compared to those in E-S0's. No relevant counterparts to these dynamically cold ICs are obtained in the remnant material originally belonging to the primary galaxy.

The geometrical analysis of these ICs reveals a wide morphological zoo of ICs, similar to the ones observed in SaSc galaxies through either direct imaging or unsharp masking, such as IDs, IRs, pseudo-rings, nested IDs, spiral structure, and different combinations of them. No discernible bars have been formed in either the primary disc or the IC, from the accreted satellite material. In addition, the structural and kinematic properties of these ICs are analogous to those observed in real galaxies. The existence of these ICs cannot be derived directly from global surface density maps and profiles, but can be deduced from their characteristic features in the isophotal profiles and kinematic maps of the final remnants, which resemble many observational cases.

The key parameters that determine whether these IDs and IRs form in our simulations, in the absence of significant bars or dissipative components, are the structure and density of the satellites, as well as the existence of a prominent bulge in the primary galaxy. Realistic satellite-to-primary galaxy density ratios help to ensure that the satellites are more sensitive to orbital circularization and disruption than those used in previous simulations.

Combined with the disc resonances induced by the encounter, these three processes (satellite disruption, orbital circularization, and coupling of disruption with merger-triggered resonances in the disc) produce highly aligned rotationallysupported ICs at the remnant centres. The existence of big bulges in the primary galaxies and long-lasting decaying orbits ensure a more efficient satellite disruption, producing thinner ICs. This implies that, if a low-density satellite (such as a dS) were accreted by a galaxy with a prominent bulge, it would result in the complete disruption of the satellite and the formation of a rotationally-supported flat IC. However, if the galaxy accreted were a high-density satellite (such as a dSph or $\mathrm{dE}$ ) or if the primary galaxy had a small bulge, the undisrupted satellite core would be expected to sink to the galaxy centre without being disrupted completely, contributing to the formation of a central bulge. In this sense, minor mergers might be the origin of old, pure stellar IDs and IRs in many unbarred galaxies (especially for counter-rotating cases).

Traditionally, minor mergers have been considered as secondary agents in the formation of dynamically-cold ICs in galaxies, that are only responsible for inducing bars in the galaxy discs in which ICs are finally produced. Our models suggest that the majority of the ICs found in spiral galaxies could have had a minor-merger related origin (independently of whether the minor merger triggers a bar or not), and that the role of minor mergers in the formation of ICs may have been far more complex than just bar triggering. In conclusion, the present models prove that minor mergers are an extremely efficient mechanism for forming rotationally-supported stellar ICs in spiral galaxies, requiring neither strong dissipation nor the development of strong bars.
Acknowledgements. We thank our anonymous referee whose suggestions helped us to improve the clarity and presentation of the results. We are also grateful to P. Erwin and L. Sparke for the permission to reproduce some figures from Erwin \& Sparke (2003), and to the AAS for providing this permission too. Supported by the Spanish Ministry of Science and Innovation (MICINN) under projects AYA2009-10368, AYA2006-12955, and AYA2009-11137, and by the Madrid Regional Government through the AstroMadrid Project (CAM S2009/ESP1496, http://www.laeff.cab.inta-csic.es/projects/astromadrid/ main/index.php). Funded by the Spanish MICINN under the ConsoliderIngenio 2010 Program grant CSD2006-00070: "First Science with the GTC" (http://www.iac.es/consolider-ingenio-gtc/). A.C.G.G. is a Ramon y Cajal Fellow of the Spanish MICINN.

\section{References}

Abadi, M. G., Navarro, J. F., Steinmetz, M., \& Eke, V. R. 2003, ApJ, 597, 21 Aguerri, J. A. L., \& González-García, A. C. 2009, A\&A, 494, 891

Aguerri, J. A. L., Balcells, M., \& Peletier, R. F. 2001, A\&A, 367, 428

Arnaboldi, M., Capaccioli, M., Cappellaro, E., Held, E. V., \& Koribalski, B. 1995a, AJ, 110, 199

Arnaboldi, M., Freeman, K. C., Sackett, P. D., Sparke, L. S., \& Capaccioli, M. 1995b, Planet. Space Sci., 43, 1377

Athanassoula, E. 2005, MNRAS, 358, 1477

Athanassoula, E., Lambert, J. C., \& Dehnen, W. 2005, MNRAS, 363, 496

Athanassoula, E., Romero-Gómez, M., Bosma, A., \& Masdemont, J. J. 2009a, MNRAS, 400, 1706

Athanassoula, E., Romero-Gómez, M., \& Masdemont, J. J. 2009b, MNRAS, 394,67

Athanassoula, E., Romero-Gómez, M., Bosma, A., \& Masdemont, J. J. 2010, MNRAS, 407, 1433

Aumer, M., Burkert, A., Johansson, P. H., \& Genzel, R. 2010, ApJ, 719, 1230

Bagley, M., Minchev, I., \& Quillen, A. C. 2009, MNRAS, 395, 537

Balcells, M., \& González, A. C. 1998, ApJ, 505, L109

Barnes, J. E. 2001, in Gas and Galaxy Evolution, ed. J. E. Hibbard, M. Rupen, \& J. H. van Gorkom, ASP Conf. Ser., 240, 135

Barnes, J. E., \& Hernquist, L. 1996, ApJ, 471, 115

Bartošková, K., Jungwiert, B., Ebrová, I., Jílková, L., \& Křížek, M. 2011 [arXiv: 1103.2562]

Bedregal, A. G., Aragón-Salamanca, A., \& Merrifield, M. R. 2006, MNRAS, 373,1125

Bender, R. 1990, A\&A, 229, 441

Bender, R., Saglia, R. P., \& Gerhard, O. E. 1994, MNRAS, 269, 785

Bendo, G. J., \& Barnes, J. E. 2000, MNRAS, 316, 315

Berentzen, I., Shlosman, I., Martinez-Valpuesta, I., \& Heller, C. H. 2007, ApJ, 666, 189

Bertola, F., Corsini, E. M., Vega Beltrán, J. C., et al. 1999, ApJ, 519, L127

Böker, T., Falcón-Barroso, J., Schinnerer, E., Knapen, J. H., \& Ryder, S. 2008, AJ, 135, 479

Bournaud, F. 2009 [arXiv: 0909. 1812]

Bournaud, F., \& Combes, F. 2002, A\&A, 392, 83

Bournaud, F., Combes, F., \& Jog, C. J. 2004, A\&A, 418, L27

Bournaud, F., Combes, F., Jog, C. J., \& Puerari, I. 2005a, A\&A, 438, 507

Bournaud, F., Jog, C. J., \& Combes, F. 2005b, A\&A, 437, 69

Brosch, N., Kniazev, A. Y., Moiseev, A., \& Pustilnik, S. A. 2010, MNRAS, 401, 2067

Buta, R., \& Combes, F. 1996, Fund. Cosm. Phys., 17, 95

Buta, R., \& Purcell, G. B. 1998, AJ, 115, 484

Buta, R., Alpert, A. J., Cobb, M. L., Crocker, D. A., \& Purcell, G. B. 1998, AJ, 116,1142

Cappellari, M., Emsellem, E., Bacon, R., et al. 2007, MNRAS, 379, 418

Carollo, C. 2004, in HST Proposal, 10157

Carollo, C. M., Stiavelli, M., Seigar, M., de Zeeuw, P. T., \& Dejonghe, H. 2002, AJ, 123, 159

Chemin, L., \& Hernandez, O. 2009, A\&A, 499, L25

Chilingarian, I. V., Novikova, A. P., Cayatte, V., et al. 2009, A\&A, 504, 389

Cinzano, P., \& van der Marel, R. P. 1994, MNRAS, 270, 325

Combes, F., Gerin, M., Nakai, N., Kawabe, R., \& Shaw, M. A. 1992, A\&A, 259, L27

Comerón, S., Knapen, J. H., Beckman, J. E., \& Shlosman, I. 2008, A\&A, 478, 403

Comerón, S., Knapen, J. H., Beckman, J. E., et al. 2010, MNRAS, 402, 2462

Cox, T. J., Jonsson, P., Somerville, R. S., Primack, J. R., \& Dekel, A. 2008, MNRAS, 384, 386

de Lapparent, V., Baillard, A., \& Bertin, E. 2011, A\&A, 532, A75

de Lorenzo-Cáceres, A., Falcón-Barroso, J., Vazdekis, A., \& MartínezValpuesta, I. 2008, ApJ, 684, L83

Di Matteo, P., Combes, F., Melchior, A., \& Semelin, B. 2008, A\&A, 477, 437 
Domínguez-Palmero, L., \& Balcells, M. 2008, A\&A, 489, 1003

Domínguez-Palmero, L., Balcells, M., Erwin, P., et al. 2008, A\&A, 488, 1167

Ebrova, I., Bartoskova, K., Jungwiert, B., Jilkova, L., \& Krizek, M. 2011 [arXiv: 1103.2565]

Eliche-Moral, M. C., Balcells, M., Aguerri, J. A. L., \& González-García, A. C. 2006, A\&A, 457, 91 (EM06)

Eliche-Moral, M. C., Prieto, M., Gallego, J., et al. 2010a, A\&A, 519, A55

Eliche-Moral, M. C., Prieto, M., Gallego, J., \& Zamorano, J. 2010b [arXiv: 1003.0686]

Elmegreen, D. M., Elmegreen, B. G., Combes, F., \& Bellin, A. D. 1992, A\&A, 257,17

Emsellem, E., Cappellari, M., Peletier, R. F., et al. 2004, MNRAS, 352, 721

Emsellem, E., Cappellari, M., Krajnović, D., et al. 2007, MNRAS, 379, 401

Erwin, P. 2004, A\&A, 415, 941

Erwin, P., \& Sparke, L. S. 2002, AJ, 124, 65

Erwin, P., \& Sparke, L. S. 2003, ApJS, 146, 299

Erwin, P., Beltrán, J. C. V., Graham, A. W., \& Beckman, J. E. 2003, ApJ, 597, 929

Falcón-Barroso, J., Balcells, M., Peletier, R. F., \& Vazdekis, A. 2003, A\&A, 405, 455

Falcón-Barroso, J., Peletier, R. F., Emsellem, E., et al. 2004, MNRAS, 350, 35

Falcón-Barroso, J., Bacon, R., Bureau, M., et al. 2006, MNRAS, 369, 529 (F06)

Faúndez-Abans, M., Fernandes, I. F., de Oliveira-Abans, M., Poppe, P. C. R., \& Martin, V. A. F. 2009, A\&A, 507, 1303

Fisher, D. B., \& Drory, N. 2010, ApJ, 716, 942

Franx, M., \& Illingworth, G. D. 1988, ApJ, 327, L55

González-García, A. C., \& Balcells, M. 2005, MNRAS, 357, 753

González-García, A. C., Balcells, M., \& Olshevsky, V. S. 2006, MNRAS, 372, L78

González-García, A. C., Oñorbe, J., Domínguez-Tenreiro, R., \& Gómez-Flechoso, M. Á. 2009, A\&A, 497, 35

Graham, A. W. 2001, AJ, 121, 820

Gutiérrez, C. M., López-Corredoira, M., Prada, F., \& Eliche, M. C. 2002, ApJ, 579,592

Haynes, M. P., Jore, K. P., Barrett, E. A., Broeils, A. H., \& Murray, B. M. 2000, AJ, 120, 703

Henriques, B. M. B., \& Thomas, P. A. 2010, MNRAS, 403, 768

Hernquist, L., \& Barnes, J. E. 1991, Nature, 354, 210

Hopkins, P. F., Bundy, K., Croton, D., et al. 2010, ApJ, 715, 202

Jesseit, R., Naab, T., Peletier, R. F., \& Burkert, A. 2007, MNRAS, 376, 997

Jogee, S., Miller, S. H., Penner, K., et al. 2009, ApJ, 697, 1971

Jungwiert, B., Combes, F., \& Axon, D. J. 1997, A\&AS, 125, 479

Kannappan, S. J., \& Fabricant, D. G. 2001, AJ, 121, 140

Kaviraj, S., Peirani, S., Khochfar, S., Silk, J., \& Kay, S. 2009, MNRAS, 394, 1713

King, I. R. 1966, AJ, 71, 64

Knapen, J. H. 2005, A\&A, 429, 141

Knapen, J. H., Whyte, L. F., de Blok, W. J. G., \& van der Hulst, J. M. 2004, A\&A, 423, 481

Knapen, J. H., Mazzuca, L. M., Böker, T., et al. 2006, A\&A, 448, 489

Knierman, K. A. 2010, in ASP Conf. Ser. 423, ed. B. Smith, J. Higdon, S. Higdon, \& N. Bastian, 342

Koprolin, W., \& Zeilinger, W. W. 2000, A\&AS, 145, 71

Kormendy, J., \& Kennicutt, Jr., R. C. 2004, ARA\&A, 42, 603

Kormendy, J., Dressler, A., Byun, Y. I., et al. 1994, in European Southern Observatory Conference and Workshop Proceedings, ed. G. Meylan, \& P. Prugniel, 49, 147

Kormendy, J., Gebhardt, K., Fisher, D. B., et al. 2005, AJ, 129, 2636

Kormendy, J., Cornell, M. E., Block, D. L., Knapen, J. H., \& Allard, E. L. 2006, ApJ, 642, 765

Krajnović, D., \& Jaffe, W. 2004, A\&A, 428, 877

Krajnović, D., Bacon, R., Cappellari, M., et al. 2008, MNRAS, 390, 93

Krajnovic, D., Emsellem, E., Cappellari, M., et al. 2011, MNRAS, 414, 2923

Kuijken, K., \& Dubinski, J. 1994, MNRAS, 269, 13

Kuijken, K., \& Dubinski, J. 1995, MNRAS, 277, 1341

Lauer, T. R., Ajhar, E. A., Byun, Y., et al. 1995, AJ, 110, 2622

Lisker, T., Debattista, V. P., Ferreras, I., \& Erwin, P. 2006, MNRAS, 370, 477

López-Sanjuan, C., Balcells, M., García-Dabó, C. E., et al. 2009, ApJ, 694, 643

López-Sanjuan, C., Balcells, M., Pérez-González, P. G., et al. 2010a, A\&A, 518, A20

López-Sanjuan, C., Balcells, M., Pérez-González, P. G., et al. 2010b, ApJ, 710, 1170
Martel, A. R., Baum, S. A., Sparks, W. B., et al. 2002, New Astron. Rev., 46, 187

Martínez-Delgado, D., Peñarrubia, J., Jurić, M., Alfaro, E. J., \& Ivezić, Z. 2007, ApJ, 660, 1264

Martínez-Delgado, D., Peñarrubia, J., Gabany, R. J., et al. 2008, ApJ, 689, 184 Martínez-Delgado, D., Pohlen, M., Gabany, R. J., et al. 2009, ApJ, 692, 955

Martínez-Delgado, D., Gabany, R. J., Crawford, K., et al. 2010, AJ, 140, 962

Martinez-Valpuesta, I., Shlosman, I., \& Heller, C. 2006, ApJ, 637, 214

Mastropietro, C., Moore, B., Mayer, L., et al. 2005, MNRAS, 364, 607

Mazzuca, L. M., Sarzi, M., Knapen, J. H., Veilleux, S., \& Swaters, R. 2006, ApJ, 649, L79

Moiseev, A. V., \& Bizyaev, D. V. 2009, New Astron. Rev., 53, 169

Moiseev, A., Sil'Chenko, O., \& Katkov, I. 2010, in AIP Conf. Ser. 1240, ed. V. P. Debattista, \& C. C. Popescu, 251

Morelli, L., Halliday, C., Corsini, E. M., et al. 2004, MNRAS, 354, 753

Morelli, L., Cesetti, M., Corsini, E. M., et al. 2010, A\&A, 518, A32

Moster, B. P., Macciò, A. V., Somerville, R. S., Johansson, P. H., \& Naab, T. 2010, MNRAS, 403, 1009

Muñoz-Tuñón, C., Caon, N., \& Aguerri, J. A. L. 2004, AJ, 127, 58

Naab, T., \& Burkert, A. 2001, ApJ, 555, L91

Naab, T., \& Burkert, A. 2003, ApJ, 597, 893

Naab, T., Khochfar, S., \& Burkert, A. 2006, ApJ, 636, L81

Norman, C. A., Sellwood, J. A., \& Hasan, H. 1996, ApJ, 462, 114

Okumura, S. K., Kawabe, R., Ishiguro, M., \& Ishizuki, S. 1994, in Astronomy with Millimeter and Submillimeter Wave Interferometry, ed. M. Ishiguro, \& J. Welch, IAU Coll., 140, ASP Conf. Ser., 59, 376

Peletier, R. F., \& Balcells, M. 1996, AJ, 111, 2238

Peletier, R. F., Falcón-Barroso, J., Bacon, R., et al. 2007, MNRAS, 379, 445

Pérez-Gallego, J., Guzmán, R., Castillo-Morales, A., et al. 2010, MNRAS, 402, 1397

Pfenniger, D., \& Norman, C. 1990, ApJ, 363, 391

Pizzella, A., Corsini, E. M., Morelli, L., et al. 2002, ApJ, 573, 131

Prieto, M., Aguerri, J. A. L., Varela, A. M., \& Muñoz-Tuñón, C. 2001, A\&A, 367,405

Regan, M. W., \& Teuben, P. 2003, ApJ, 582, 723

Reshetnikov, V., Bournaud, F., Combes, F., et al. 2005, A\&A, 431, 503

Rest, A., van den Bosch, F. C., Jaffe, W., et al. 2001, AJ, 121, 2431

Riffel, R. A. 2010, Ap\&SS, 327, 239

Romero-Gómez, M., Athanassoula, E., Masdemont, J. J., \& García-Gómez, C. 2007, A\&A, 472, 63

Roškar, R., Debattista, V. P., Brooks, A. M., et al. 2010, MNRAS, 408, 783

Sarzi, M., Falcón-Barroso, J., Davies, R. L., et al. 2006, MNRAS, 366, 115

Sarzi, M., Allard, E. L., Knapen, J. H., \& Mazzuca, L. M. 2007, MNRAS, 380, 949

Scorza, C., \& van den Bosch, F. C. 1998, MNRAS, 300, 469

Scorza, C., Bender, R., Winkelmann, C., Capaccioli, M., \& Macchetto, D. F. 1998, A\&AS, 131, 265

Sellwood, J. A. 2010 [arXiv: 1006. 4855]

Shapiro, K. L., Falcón-Barroso, J., van de Ven, G., et al. 2010, MNRAS, 402, 2140

Shu, F. H. 1969, ApJ, 158, 505

Sil'Chenko, O. 2010, in AIP Conf. Ser. 1240, ed. V. P. Debattista, \& C. C. Popescu, 255

Sil'Chenko, O. K., \& Moiseev, A. V. 2006, AJ, 131, 1336

Sil'Chenko, O. K., \& Smirnova, A. A. 2010, Astron. Lett., 36, 319

Sil'Chenko, O. K., Chilingarian, I. V., Sotnikova, N. Y., \& Afanasiev, V. L. 2011 MNRAS, 414, 3645

Springel, V. 2005, MNRAS, 364, 1105

Springel, V., Yoshida, N., \& White, S. D. M. 2001, New Astron., 6, 79

Tapia, M. T., Balcells, M., \& Eliche-Moral, M. C. 2010a, in IAU Symp. 262, ed. G. Bruzual, \& S. Charlot, 432

Tapia, M. T., Balcells, M., \& Eliche-Moral, M. C. 2010b, in AIP Conf. Ser. 1240, ed. V. P. Debattista, \& C. C. Popescu, 423

Thakar, A. R., \& Ryden, B. S. 1996, ApJ, 461, 55

Thakar, A. R., \& Ryden, B. S. 1998, ApJ, 506, 93

Thakar, A. R., Ryden, B. S., Jore, K. P., \& Broeils, A. H. 1997, ApJ, 479, 702

Tully, R. B., \& Fisher, J. R. 1977, A\&A, 54, 661

van den Bosch, F. C., \& Emsellem, E. 1998, MNRAS, 298, 267

van den Bosch, F. C., Ferrarese, L., Jaffe, W., Ford, H. C., \& O'Connell, R. W. 1994, AJ, 108, 1579

van den Bosch, F. C., Jaffe, W., \& van der Marel, R. P. 1998, MNRAS, 293, 343 van der Marel, R. P., \& Franx, M. 1993, ApJ, 407, 525 
a) M6PsDb: Nested IDs

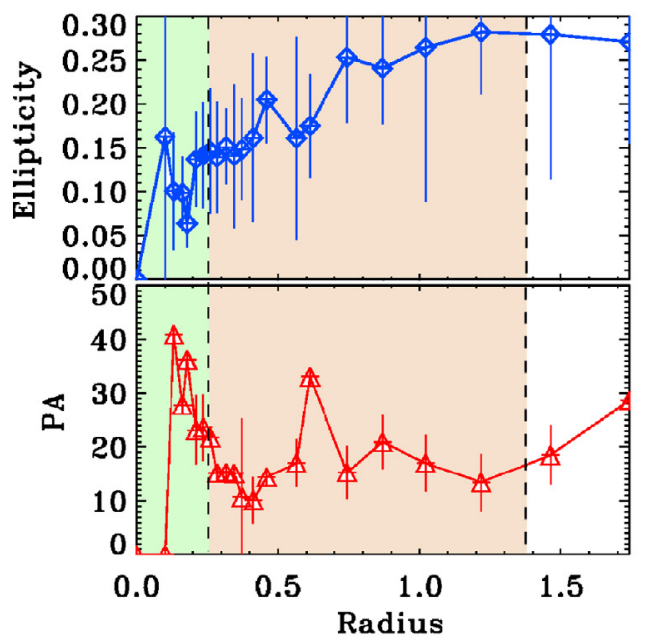

b) M6PsRb: Nested IDs

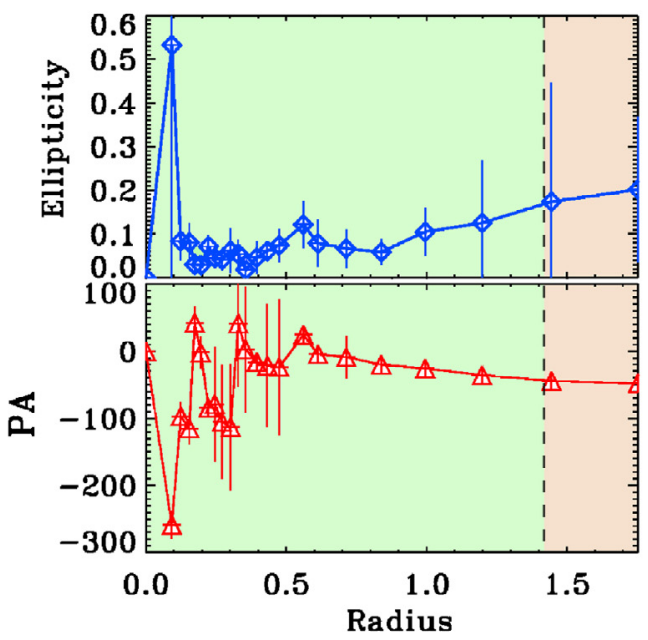

c) M6PIDb: Nested IDs

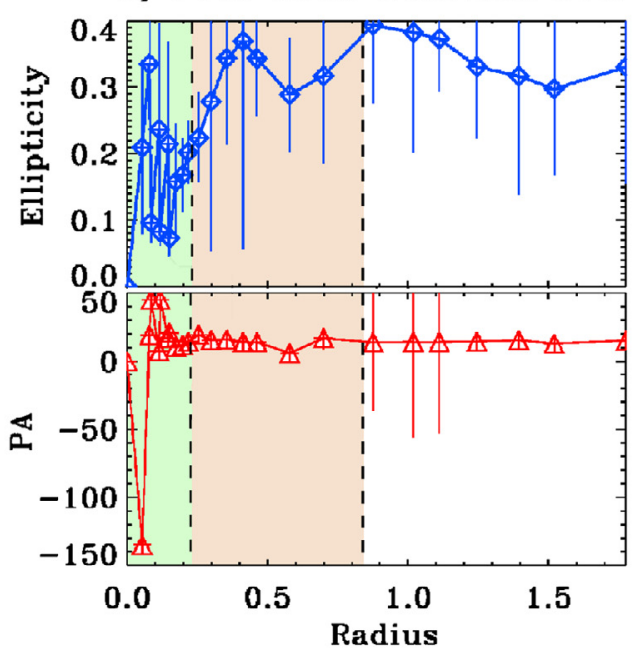

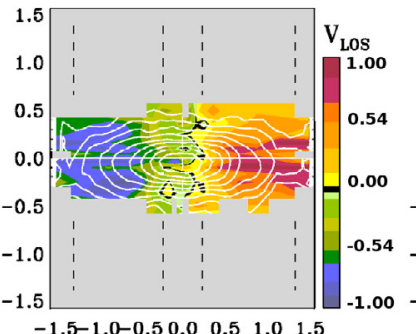
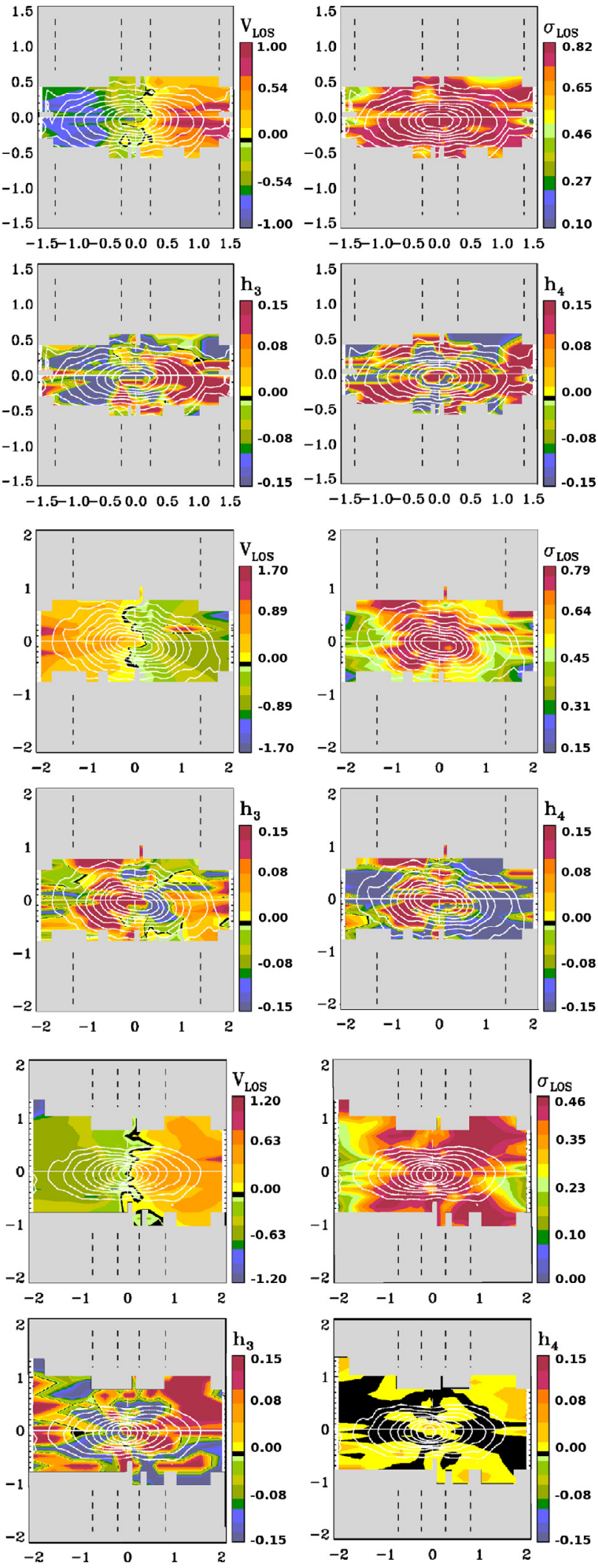

Fig. 12. Photometrical and kinematical features imprinted by the formed ICs in the global stellar maps of the final remnants of models M6Ps[D/R $]$ b and M6PIDb (models a to $\mathrm{c}$ in Table 3). Left panels: ellipticity and PA isophotal profiles of all the stars in the remnants, using an inclined view $\left(\theta=60^{\circ}, \phi=20^{\circ}\right)$. The extent of the ICs as defined in Sect. 3.2.1 is indicated by vertical dashed lines in each case for reference (accordingly to Fig. 2). Right panels: $2 \mathrm{D}$-maps of the kinemetric moments of the LOSVD of all the stars in the remnant using an edge-on view ( $\left.V_{\mathrm{LOS}}, \sigma, h_{3}, h_{4}\right)$. The levels are distributed linearly (simulation units). The photometric axes defined by the material in the core region of the galaxy are also plotted (straight lines). The isophotes of the stellar material in the remnant originally belonging to the satellite are overplotted for reference (contours). 
M. C. Eliche-Moral et al.: A minor merger origin for stellar inner discs and rings in spiral galaxies

d) M6PIRb: IR + ID

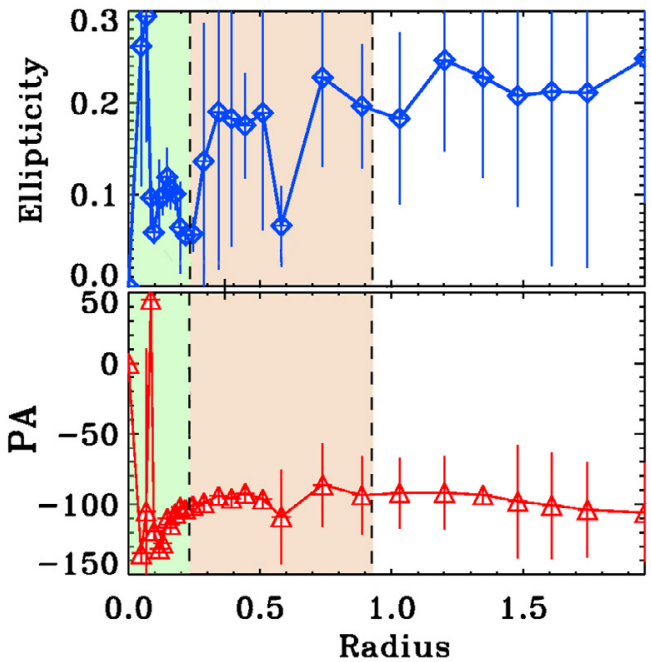

e) M6PsDs: Bulge + ID

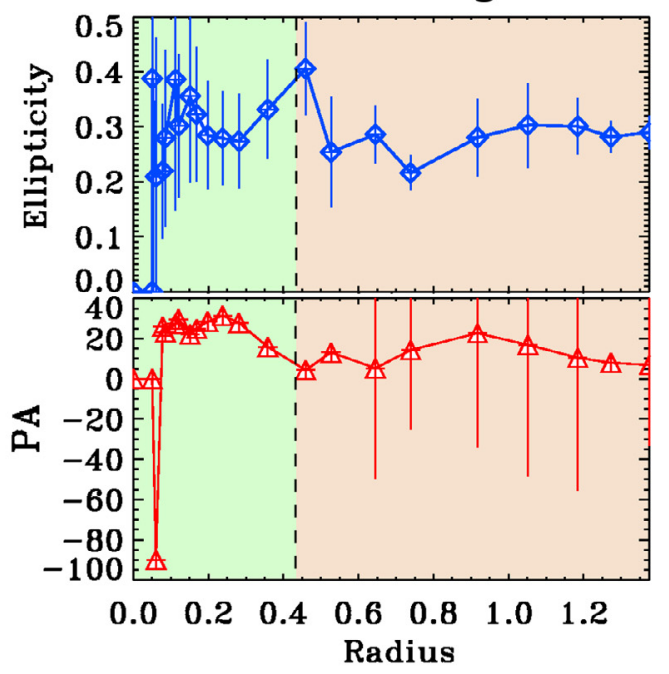

f) M6PsRs: Bulge + ID

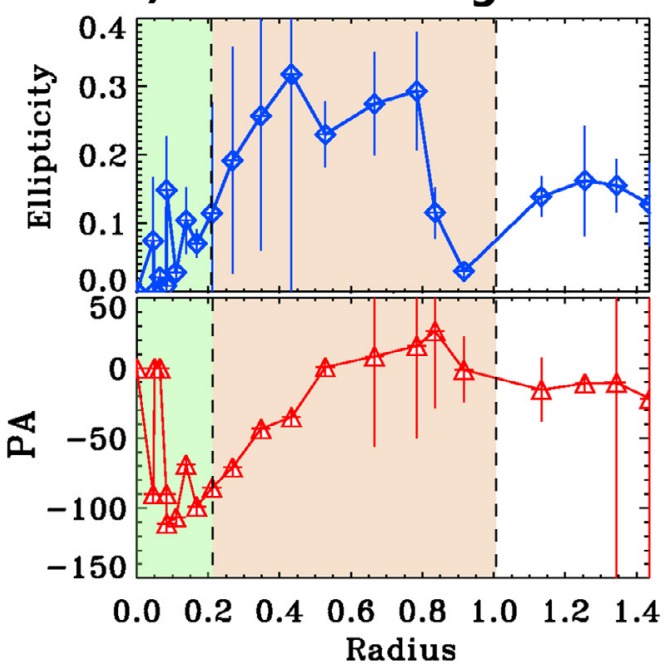

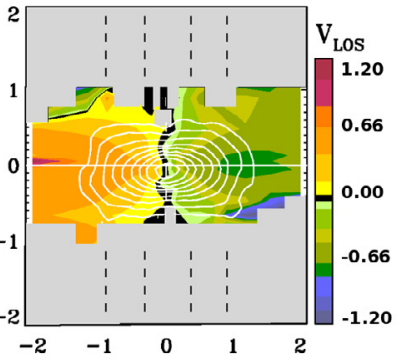
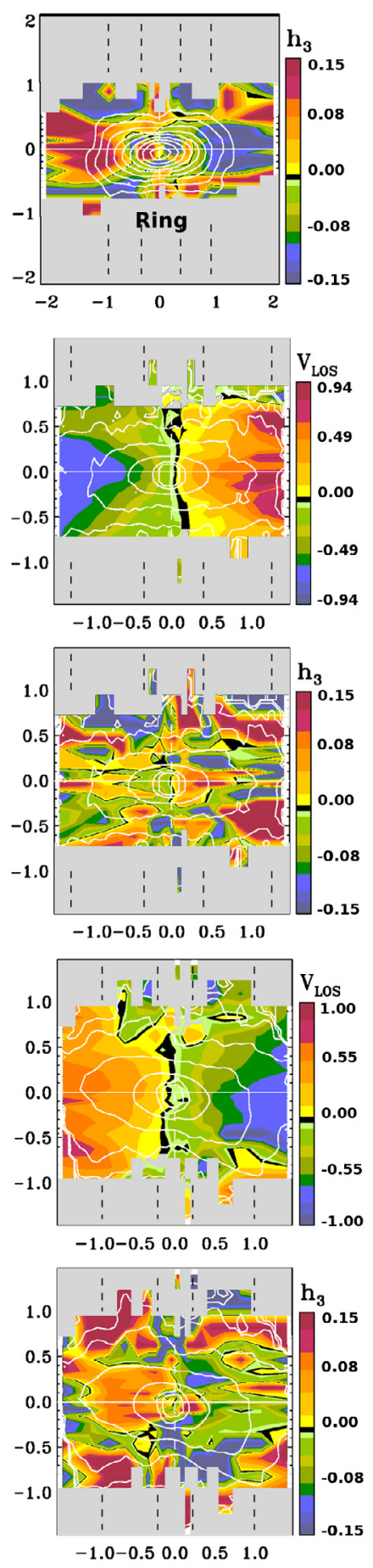
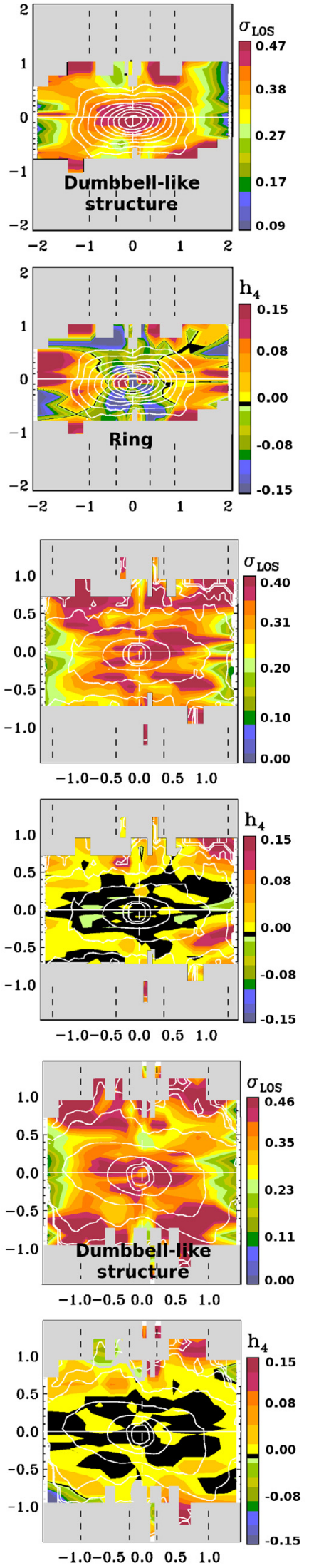

Fig. 13. Photometrical and kinematical features produced by the ICs formed, in the global stellar maps of the final remnants of models M6PIRb and M6Ps[D/R]s (models d to $\mathrm{f}$ in Table 3). See caption of Fig. 12. 


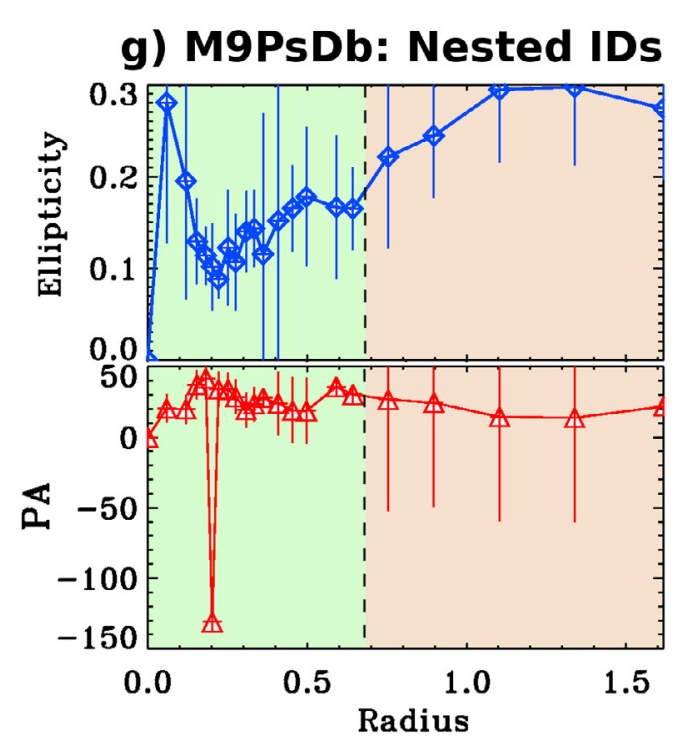

h) M9PsRb: ID

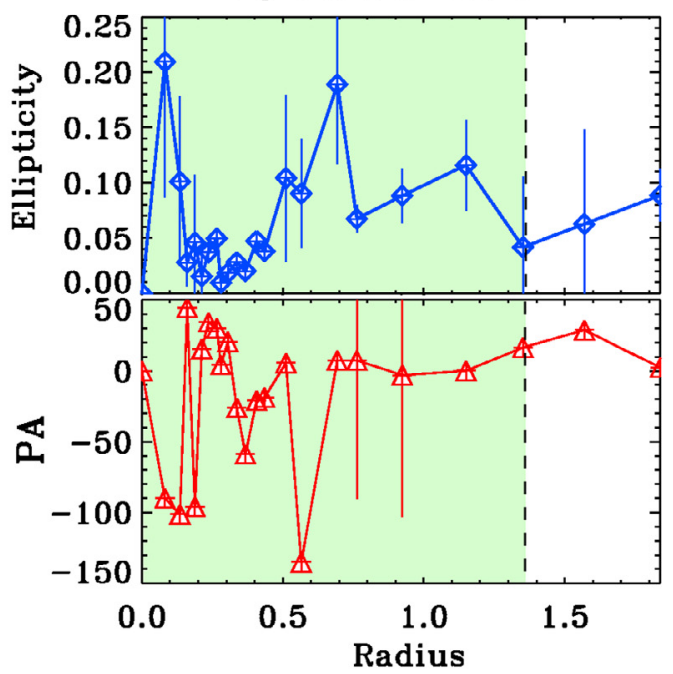

i) M18PsDb: IR + ID

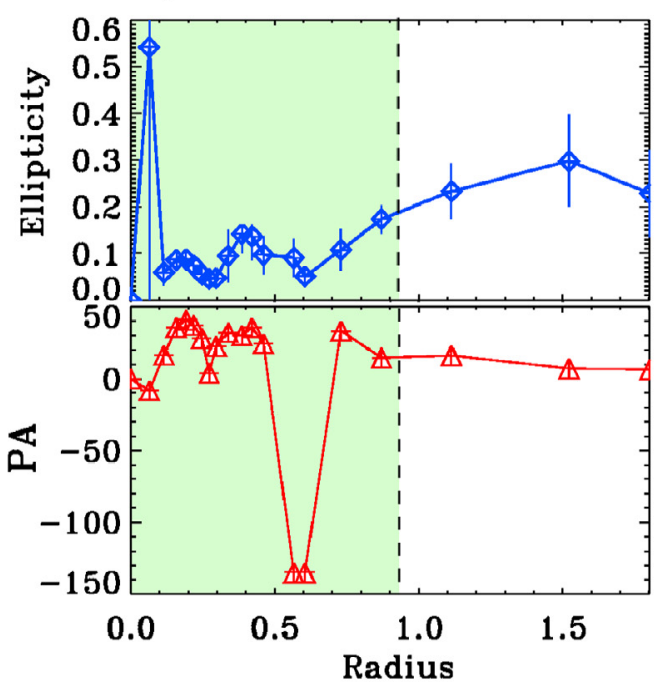

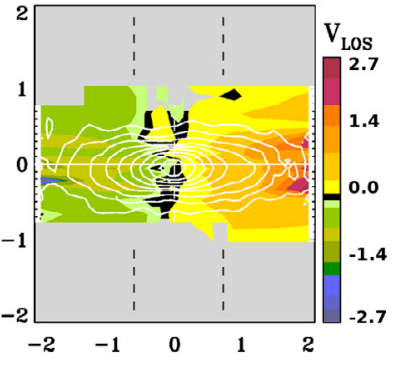
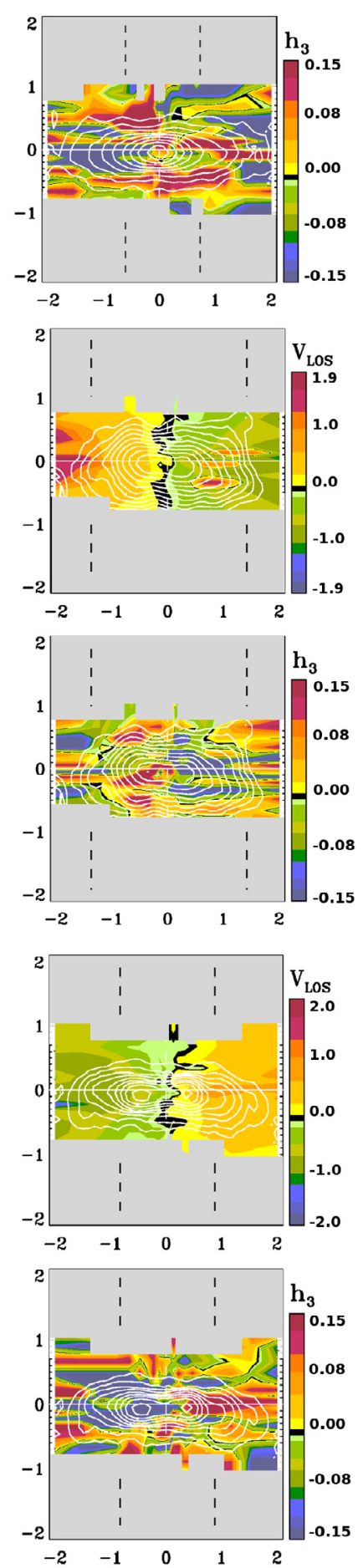
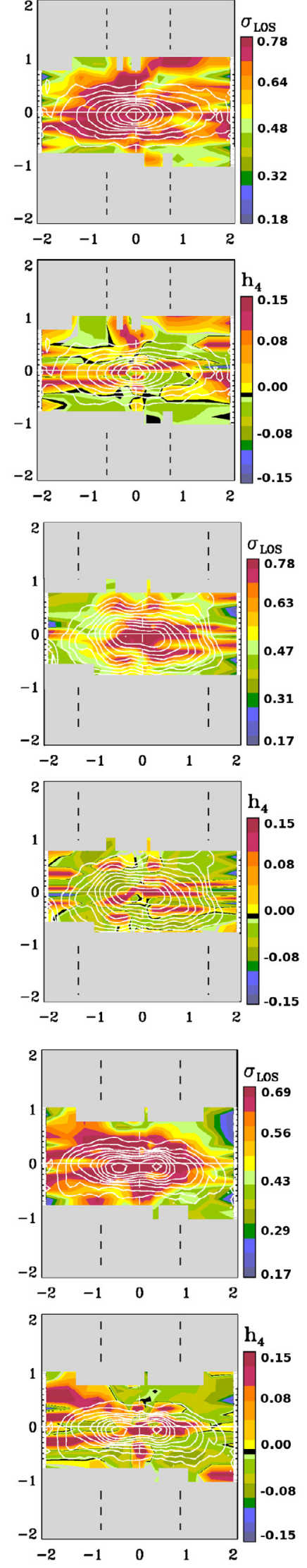

Fig. 14. Photometrical and kinematical features produced by the ICs formed, in the global stellar maps of the final remnants of models M9Ps[D/R $] b$ and M18PsDb (models g to i in Table 3). See caption of Fig. 12. 
M. C. Eliche-Moral et al.: A minor merger origin for stellar inner discs and rings in spiral galaxies
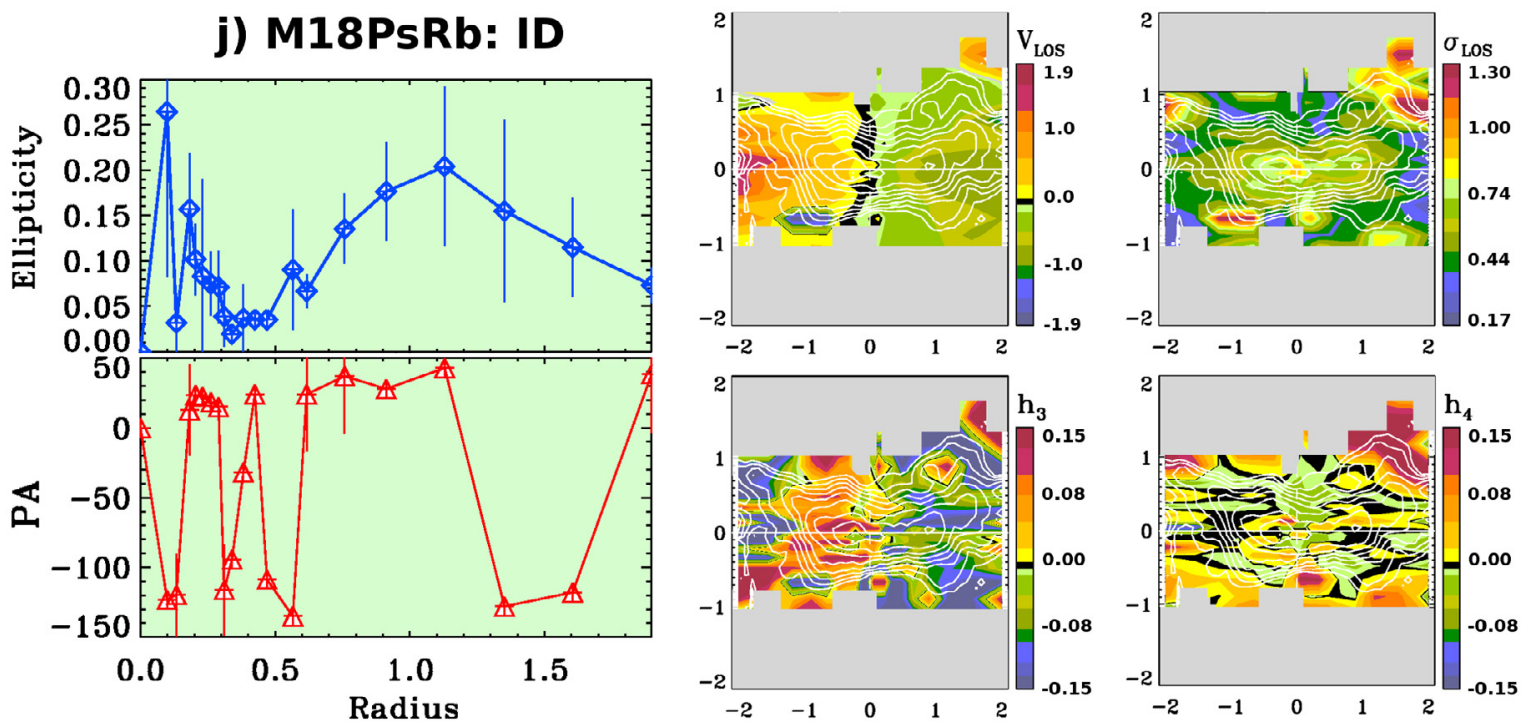

k) M18PIDb: Nested IDs
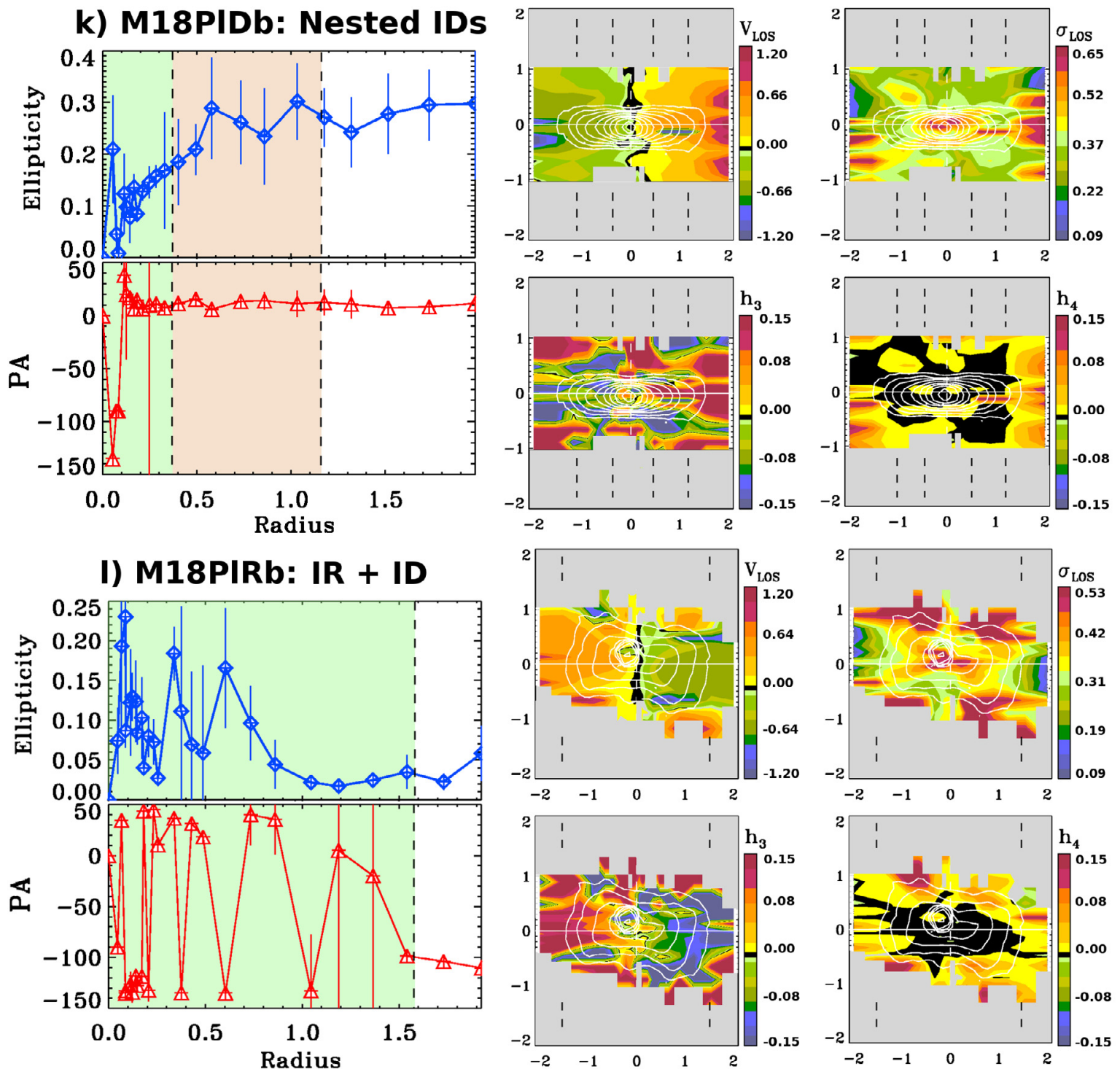

Fig. 15. Photometrical and kinematical features produced by the ICs formed, in the global stellar maps of the final remnants of models M18PsRb and M18Pl[D/R]b (models j to 1 in Table 3). See caption of Fig. 12. 
MODELLED ICS

b) M6PsRb

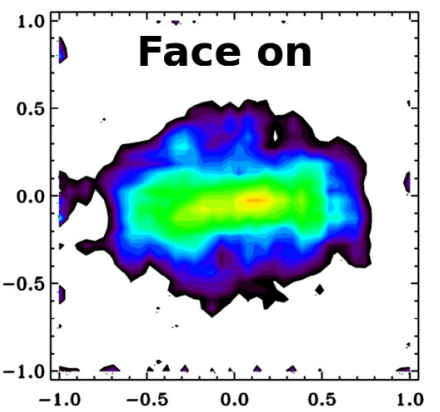

g) M9PsDb

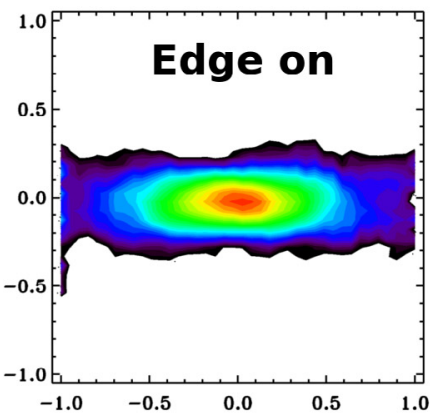

i) M18PsDb

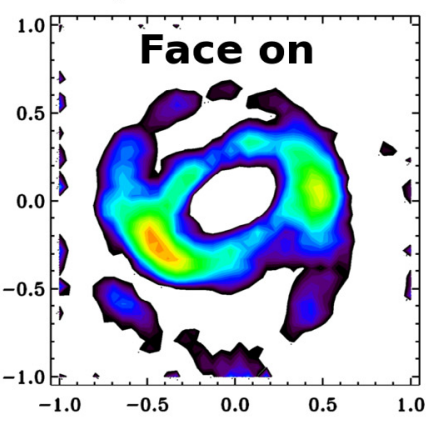

k) M18PIDb

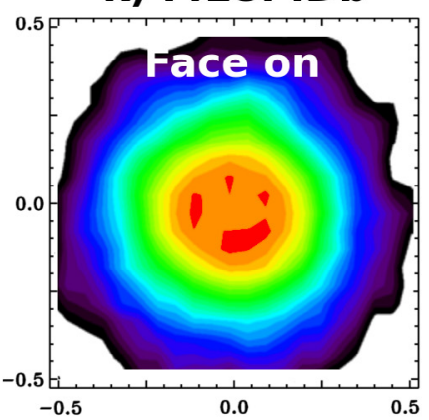

REAL ICs

NGC 4665

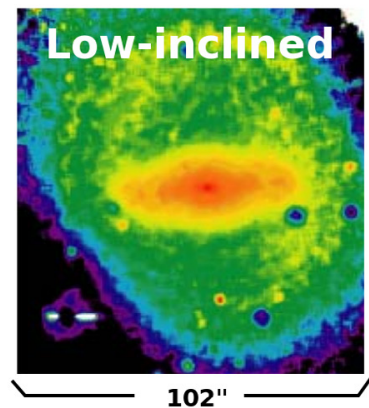

NGC 5377

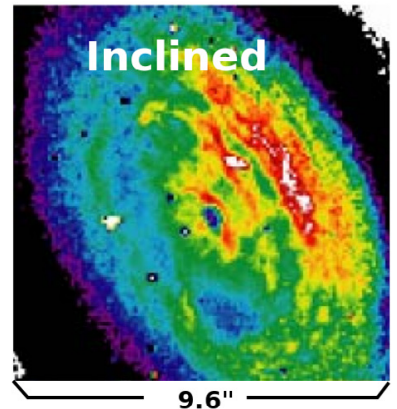

NGC 4314

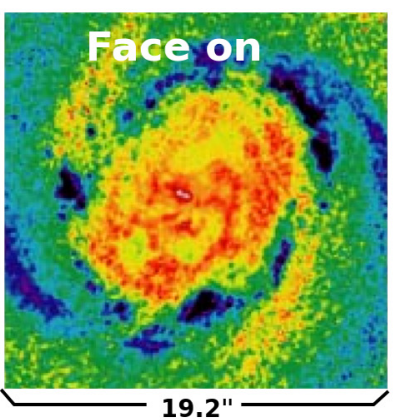

NGC 4245

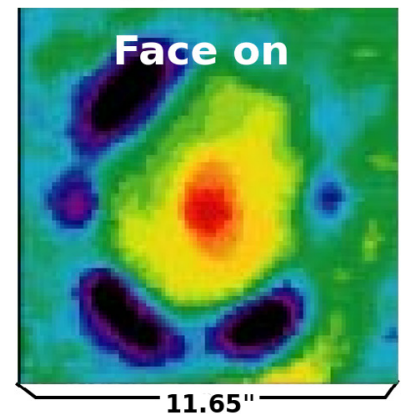

MODELLED ICS

d) M6PIRb

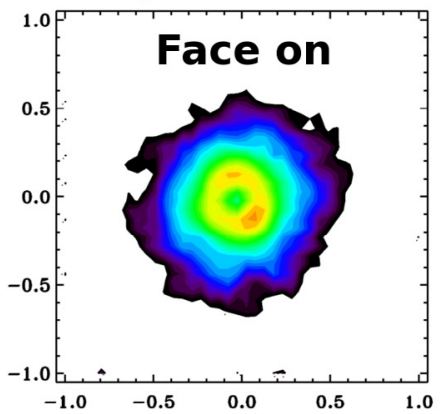

h) M9PsRb

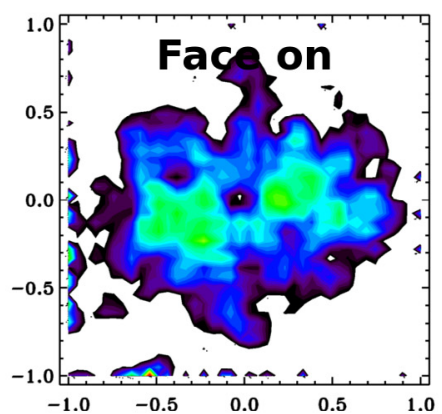

j) M18PsRb

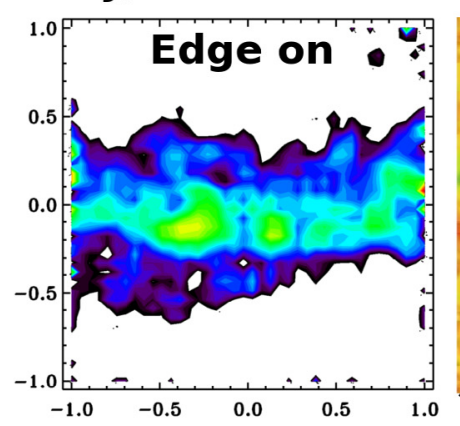

I) M18PIRb

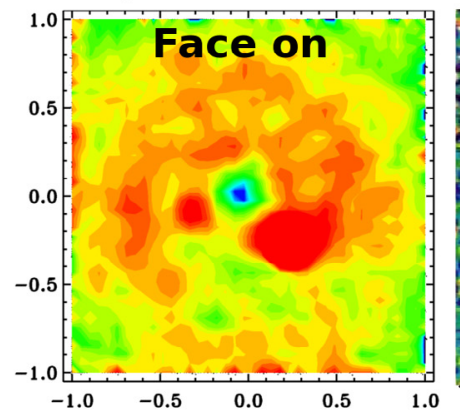

REAL ICS

NGC 2273

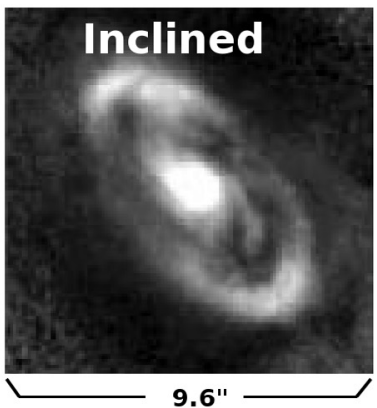

NGC 6654

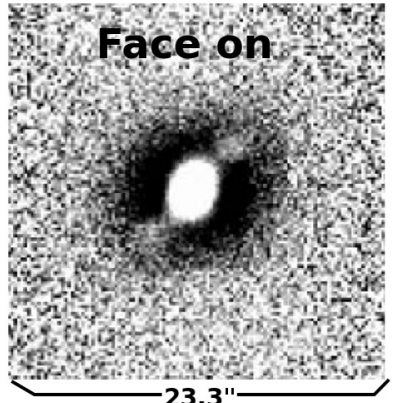

NGC 4691

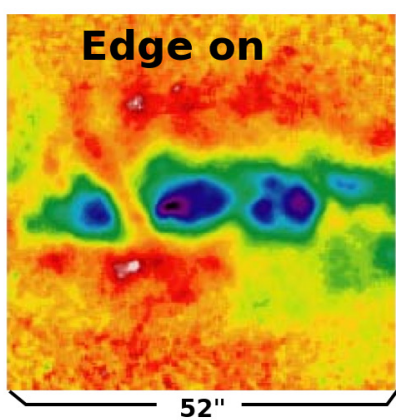

NGC 718

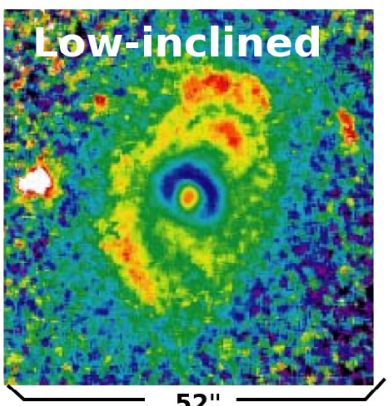

Fig. 16. Comparison of some ICs obtained in our minor merger experiments (first and third columns in the figure) to real observational examples with similar morphologies (second and fourth columns, respectively). The surface density maps of the ICs generated in our models are taken from Figs. 4, 5 and are in simulation units (consult captions there). The observational examples are taken from the sample of spiral galaxies with ICs developed by Erwin \& Sparke (2003). Coloured maps of real galaxies correspond to $B-R$ or $V-I$ colour maps of these galaxies, while their greyscale maps represent unsharp masks in $V, R$ or $H$ bands (see Erwin \& Sparke 2003 for a detailed description of each frame). The global inclination of galaxy in the observational cases and the spatial scale of each postage stamp are indicated in each frame. In images of real galaxies, north is up and east is left, except in NGC 4665, which has been rotated $90^{\circ}$ clockwise to emphasize the similarity with the IC resulting in experiment M6PsRb (model b). For a comparison of the scaled sizes of the ICs resulting in our models with those exhibited by real ICs, consult Sect. 4. Material from Erwin \& Sparke (2003) in this figure is reproduced by permission of the American Astronomical Society (AAS) and of the original authors. 